\title{
A hybrid Finite Element-Statistical Energy Analysis method for impulsive and transient loading
}

\author{
D.H. Hawes*a, R.S. Langley ${ }^{a}$, T. Butlin ${ }^{a}$, Y. Ishii ${ }^{b}$ \\ ${ }^{\text {aDepartment }}$ of Engineering, University of Cambridge \\ Trumpington Street, Cambridge, CB2 1PZ, UK
}

${ }^{b}$ Mitsubishi Heavy Industries, Ltd. 5-717-1,

Fukahori-machi, Nagasaki, 851-0392, Japan

* Corresponding author

Email address: David.HAWES@3ds.com

Present address: Dassault Systèmes, Cambridge Science Park, Cambridge, CB4 OWN, UK

\begin{abstract}
The hybrid approach coupling Statistical Energy Analysis (SEA) and the finite element method has become a prominent technique for analysing structures under steady-state loads in the 'midfrequency' range where some components behave in a deterministic manner with low modal density and others in a statistical manner with high modal density and statistical overlap. In this paper, the method is extended from its current steady-state capability to provide calculation of structural responses under impulsive and time-varying loads. Similar to the steady-state method, a system is split into deterministic components that are modelled using the finite element approach and statistical components that are modelled as SEA subsystems.

An evolutionary spectrum approach based on the Priestley description of random processes is applied to model the response of both the SEA and deterministic components which are coupled by
\end{abstract}


considering a power balance between the SEA subsystems. The diffuse field reciprocity relationship that relates the reverberant forces generated by a subsystem to the energy within it is explored under transient conditions where it is found that it can be important to account for the build-up of a reverberant field following an impulse. Results from the method are compared against finite element simulations for a system of plates coupled by a beam and it is found to generate predictions with the accuracy expected of an SEA-based method, although deterministic oscillations of the deterministic system at early times are not accounted for.

Keywords: TSEA; Mid-frequency problem; Hybrid FE-SEA; Shock-induced vibration; Priestley description

\section{Introduction}

A wide range of standard modelling techniques exist for analysing the vibrational response of engineering structures, with the most suitable method often depending on the system, frequency range and loading conditions of interest. For systems containing only a few distinct modes at the frequencies of interest, typically true at low frequencies, the finite element method (FE) is most commonly employed. Whereas, when many overlapping modes are present, typically at high frequencies, statistical approaches become more desirable since the lengthscale of vibrations approaches that of any imperfections in the system and so the frequency response of a number of nominally identical structures can diverge. Statistical Energy Analysis [1] (SEA) is a prominent method for modelling such structures where a system is split into subsystems of similar properties and the vibrational energy within each subsystem is calculated via a power balance.

Many structures, however, will contain components with distinct modal responses as well as components that behave statistically all in the same frequency range, typically in the so-called 'midfrequency' range. In order to calculate the response of such structures under steady loads, a number of methods have been proposed, a helpful summary of which can be found in [2] with more detail in [3]. Of particular interest here is the Hybrid FE-SEA method of [4] which splits the structure into deterministic components modelled with FE and statistical components modelled with SEA. A similar approach is adopted in this paper to predict the response of such structures subject to impulsive and time-varying loading.

Most commonly, structures are investigated under steady loading conditions; however, in reality transient conditions, particularly impulsive excitation occur frequently, for example hitting a bump or changing speed in a car. Few techniques exist to predict the mid-frequency transient response of 
systems and generally FE analysis is most applicable despite its computational expense. At high frequencies, transient SEA (TSEA) is commonly applied [1,5-12] and has recently been formalised [13] by using the Priestley description of random processes $[14,15]$. In this paper, the FE and TSEA methods are combined to provide the benefits of each for systems containing some components best modelled deterministically and others best modelled statistically. To represent the transient response of the structure, the Priestley description of random processes is adopted and provides evolutionary spectra of both the energy in the SEA components and the mean square displacements of the deterministic degrees of freedom.

A key component of the steady-state hybrid method of [4] is how the FE and SEA components are coupled together. To enable this, the diffuse field reciprocity relationship $[16,17]$ was developed which expresses the mean square reverberant forces from the subsystems on the deterministic system in terms of the energy of the subsystems. This allows a power balance for each subsystem to be calculated to yield the response variables. In the transient case investigated in this paper, a transient form of this relationship is required and is investigated in Section 4.1.

In what follows, the Priestley representation is briefly introduced in Section 2 and the derivation of the transient Hybrid FE-SEA method is presented in Section 3 before numerical validation of the method using FE simulations is demonstrated in Section 4.

\section{The Priestley description}

Transient methods that investigate high frequency responses are concerned with both the time and frequency content of a structure and this requires a rigorous definition. Similar to [13], the formalism introduced by Priestley $[14,15]$ to study non-stationary random vibration can be applied, where in this case it is applied to a random system instead of a random process. If $\mathbf{q}(t)$ is a $K$-dimensional vector of stationary random variables, it can be related to its Fourier transform via the expression

$$
\mathbf{q}(t)=\int_{-\infty}^{\infty} \widehat{\mathbf{q}}(\omega) \mathrm{e}^{\mathrm{i} \omega t} \mathrm{~d} \omega
$$

and the mean square correlation matrix of the variable then has form

$$
\mathrm{E}\left[\mathbf{q}(t) \mathbf{q}^{\mathrm{T}}(t)\right]=\int_{-\infty}^{\infty} \int_{-\infty}^{\infty} \mathrm{E}\left[\widehat{\mathbf{q}}(\omega) \widehat{\mathbf{q}}^{\mathrm{T} *}\left(\omega^{\prime}\right)\right] \mathrm{e}^{\mathrm{i}\left(\omega-\omega^{\prime}\right) t} \mathrm{~d} \omega \mathrm{d} \omega^{\prime}
$$

where E[..] represents the ensemble average. In order for Eq. (2) to be compatible with the singlesided cross-spectrum $\mathbf{S}_{\mathrm{qq}}(\omega)$ of $\mathbf{q}(t)$ we must have 


$$
\mathrm{E}\left[\widehat{\mathbf{q}}(\omega) \widehat{\mathbf{q}}^{\mathrm{T} *}\left(\omega^{\prime}\right)\right]=\frac{1}{2} \mathbf{S}_{\mathrm{qq}}(\omega) \delta\left(\omega-\omega^{\prime}\right), \quad \mathrm{E}\left[\mathbf{q}(t) \mathbf{q}^{\mathrm{T}}(t)\right]=\int_{0}^{\infty} \mathbf{S}_{\mathrm{qq}}(\omega) \mathrm{d} \omega
$$

Equation (1) can also be expressed as a so-called Stieltjes integral in the form

$$
\begin{gathered}
\mathbf{q}(t)=\int_{-\infty}^{\infty} \mathrm{e}^{\mathrm{i} \omega t} \mathrm{~d} \mathbf{S}(\omega), \\
\mathrm{d} \mathbf{S}(-\omega)=\mathrm{d} \mathbf{S}^{*}(\omega), \quad E\left[\mathrm{~d} \mathbf{S}(\omega) \mathrm{d} \mathbf{S}^{\mathrm{T} *}\left(\omega^{\prime}\right)\right]=\frac{1}{2} \mathbf{S}_{\mathrm{qq}}(\omega) \delta\left(\omega-\omega^{\prime}\right) \mathrm{d} \omega \mathrm{d} \omega^{\prime} .
\end{gathered}
$$

Equation (5) forms the basis of Priestley's representation of a non-stationary random process, in which the equation is generalized to

$$
\mathbf{q}(t)=\int_{-\infty}^{\infty} \mathbf{Q}(\omega, t) \mathrm{e}^{\mathrm{i} \omega t} \mathrm{~d} \mathbf{S}(\omega)
$$

where $\mathbf{Q}(\omega, t)$ is termed the evolutionary spectrum and is a deterministic $K \times K$ matrix with

$$
\mathbf{Q}(-\omega, t)=\mathbf{Q}^{*}(\omega, t)
$$

The mean square correlation matrix of $\mathbf{q}(t)$ is then time varying with

$$
\mathrm{E}\left[\mathbf{q}(t) \mathbf{q}^{\mathrm{T}}(t)\right]=\int_{0}^{\infty} \mathbf{Q}(\omega, t) \mathbf{Q}^{\mathrm{T} *}(\omega, t) \mathbf{S}_{\mathrm{qq}}(\omega) \mathrm{d} \omega
$$

Note that without loss of generality in the Priestley description we can put

$$
\mathbf{S}_{\mathrm{qq}}(\omega)=\mathbf{I} \text {, }
$$

so that $\mathbf{Q}(\omega, t)$ acts on a vector of independent white noise variables.

\section{Methodology}

\subsection{Equations of motion}

This subsection derives the equations of motion for a hybrid FE-SEA system. As described in [4] for the steady-state hybrid approach[4], a system can be split into deterministic components with deterministic degrees of freedom, denoted with the vector $\mathbf{q}(t)$, and statistical components whose responses are characterised by their energies, denoted with the vector $\mathbf{E}(t)$. The deterministic degrees of freedom can be considered using the Priestley notation to take the form of Eq. (8) and if the deterministic system has mass, damping and stiffness matrices $\mathbf{M}, \mathbf{C}$ and $\mathbf{K}$ then its equation of motion can be written as

$$
\mathbf{M} \ddot{\mathbf{q}}+\mathbf{C} \dot{\mathbf{q}}+\mathbf{K q}=\mathbf{f}
$$


where $\mathbf{f}$ is the external force acting on the deterministic system. Using the Priestley notation for the response and forcing term by substituting Eq. (8) into Eq. (12) yields

$$
\int_{-\infty}^{\infty}\left[\mathbf{M} \frac{\mathrm{d}^{2}}{\mathrm{~d} t^{2}}\left(\mathbf{Q} \mathrm{e}^{\mathrm{i} \omega t}\right)+\mathbf{C} \frac{\mathrm{d}}{\mathrm{d} t}\left(\mathbf{Q} \mathrm{e}^{\mathrm{i} \omega t}\right)+\mathbf{K} \mathbf{Q} \mathrm{e}^{\mathrm{i} \omega t}\right] \mathrm{d} \mathbf{S}(\omega)=\int_{-\infty}^{\infty} \mathbf{F} \mathrm{e}^{\mathrm{i} \omega t} \mathrm{~d} \mathbf{S}(\omega)
$$

where $\mathbf{F}=\mathbf{F}(\omega, t)$ is the evolutionary spectrum of the force vector, $\mathbf{f}$, defined using Eq. (8) and the arguments have been omitted for brevity. Multiplying Eq. (13) by $\mathrm{d} \mathbf{S}^{* \mathrm{~T}}\left(\omega^{\prime}\right)$ then integrating over the entire $\omega^{\prime}$ domain and taking the ensemble average yields

$$
\mathbf{M} \frac{\mathrm{d}^{2}}{\mathrm{~d} t^{2}}\left(\mathbf{Q} \mathrm{e}^{\mathrm{i} \omega t}\right)+\mathbf{C} \frac{\mathrm{d}}{\mathrm{d} t}\left(\mathbf{Q} \mathrm{e}^{\mathrm{i} \omega t}\right)+\mathbf{K} \mathbf{Q} \mathrm{e}^{\mathrm{i} \omega t}=\mathbf{F} \mathrm{e}^{\mathrm{i} \omega t}
$$

A similar equation can be found for the connection to each subsystem, although the properties of each subsystem will vary over an ensemble due to variations between components. Under steady harmonic loading at frequency $\omega$, the equations of motion of a single realisation of the $j$ th subsystem can be represented by the dynamic stiffness matrix, $\mathbf{D}^{(j)}(\omega)$, of the boundary degrees of freedom that connect it to the deterministic system such that

$$
\mathbf{D}^{(j)}(\omega) \mathbf{q}(\omega)=\mathbf{f}^{(j)}(\omega)
$$

Here $\mathbf{f}^{(j)}$ is the force acting on the subsystem from the deterministic system and $\mathbf{D}^{(j)}$ relates only to the degrees of freedom of $\mathbf{q}$ that correspond to the coupling to the jth subsystem and is padded with zeros elsewhere.

In the transient case, the dynamic stiffness approach is no longer valid and an approximate representation is required that describes the subsystem in terms of mass, damping and stiffness matrices, $\mathbf{M}^{(j)}(\omega), \mathbf{C}^{(j)}(\omega)$ and $\mathbf{K}^{(j)}(\omega)$ respectively such that

$$
\mathbf{M}^{(j)}(\omega) \ddot{\mathbf{q}}(t)+\mathbf{C}^{(j)}(\omega) \dot{\mathbf{q}}(t)+\mathbf{K}^{(j)}(\omega) \mathbf{q}(t)=\mathbf{f}^{(j)}(t)
$$

where $-\omega^{2} \mathbf{M}^{(j)}+\mathrm{i} \omega \mathbf{C}^{(j)}+\mathbf{K}^{(j)}=\mathbf{D}^{(j)}$. Here, Eq. (16) is to be solved at a fixed frequency, such that it represents the time response of the deterministic system when the mass, damping and stiffness matrices of the subsystem are set to provide the dynamic stiffness found under harmonic excitation at frequency $\omega$. Provided that the response can be considered as approximately steady and harmonic, then Eq. (16) will provide a good approximation. This will be true when the envelope of the response varies slowly with time compared to the time period of the oscillations. Using the method of [18] which ensures that the mass matrix is related to the kinetic energy of the system, the matrices can be calculated as $\mathbf{M}^{(j)}(\omega)=\operatorname{Re}\left[\mathrm{dD}^{(j)}(\omega) / \mathrm{d} \omega\right] /(-2 \omega), \quad \mathbf{C}^{(j)}(\omega)=\operatorname{Im}\left[\mathbf{D}^{(j)}(\omega) / \omega\right]$ and $\mathbf{K}^{(j)}(\omega)=$ $\operatorname{Re}\left[\mathbf{D}^{(j)}(\omega)\right]+\omega^{2} \mathbf{M}^{(j)}(\omega)$. 
The force from any one realisation of a subsystem can be considered as a combination of the subsystem's direct field, denoted with the subscript 'dir', and a random reverberant force $\mathbf{f}_{\text {rev }}^{(j)}$ from the reverberant field. Following the same process as Eqs. (12)-(13) to convert to the evolutionary spectrum notation and using the naming convention that the evolutionary spectrum of a variable is denoted using the upper case, Eq. (16) can then be rewritten in the same form as Eq. (14) as

$$
\mathbf{M}_{\text {dir }}^{(j)}(\omega) \frac{\mathrm{d}^{2}}{\mathrm{~d} t^{2}}\left(\mathbf{Q} \mathrm{e}^{\mathrm{i} \omega t}\right)+\mathbf{C}_{\text {dir }}^{(j)}(\omega) \frac{\mathrm{d}}{\mathrm{d} t}\left(\mathbf{Q} \mathrm{e}^{\mathrm{i} \omega t}\right)+\mathbf{K}_{\mathrm{dir}}^{(j)}(\omega) \mathbf{Q} \mathrm{e}^{\mathrm{i} \omega t}=\mathbf{F}^{(j)} \mathrm{e}^{\mathrm{i} \omega t}+\mathbf{F}_{\mathrm{rev}}^{(j)} \mathrm{e}^{\mathrm{i} \omega t}
$$

where $\mathbf{M}_{\mathrm{dir}}^{(j)}(\omega), \mathbf{C}_{\mathrm{dir}}^{(j)}(\omega)$ and $\mathbf{K}_{\mathrm{dir}}^{(j)}(\omega)$ are the direct field mass, damping and stiffness matrices of the subsystem. These matrices are analogous to the direct field dynamic stiffness matrix, $\mathbf{D}_{\text {dir }}^{(j)}(\omega)$, used in the steady-state hybrid FE-SEA method [4] and found by considering the subsystem extended infinitely without boundaries [16]. They can be calculated from $\mathbf{D}_{\text {dir }}^{(j)}(\omega)$ using the method described above and occur as frequency dependent properties within a differential equation meaning Eq. (17) must be evaluated over time at a given frequency.

For some combinations of a deterministic system and SEA subsystems, for example a plate with a spring connection, neither the direct field response nor the deterministic system will contain a mass dependent term and Eq. (17) will be first order. Consequently, a first order analysis of the equations becomes more general and can be undertaken by setting Eq. (14) and (if applicable) (17) in state-space form. The deterministic system can be coupled to the SEA subsystems by combining Eqs. (14) and (17) and setting in state-space form producing

$$
\boldsymbol{\Gamma}_{\text {tot }}(\omega) \frac{\mathrm{d}}{\mathrm{d} t}\left(\boldsymbol{\Lambda}(\omega, \mathrm{t}) \mathrm{e}^{\mathrm{i} \omega t}\right)+\mathbf{G}_{\text {tot }}(\omega) \boldsymbol{\Lambda}(\omega, \mathrm{t}) \mathrm{e}^{\mathrm{i} \omega t}=\mathbf{U}(\omega, \mathrm{t}) \mathrm{e}^{\mathrm{i} \omega t}
$$

where if $N$ is the number of subsystems

$$
\boldsymbol{\Gamma}_{\mathrm{tot}}=\left[\begin{array}{cc}
\mathbf{I} & \mathbf{0} \\
\mathbf{0} & \mathbf{M}
\end{array}\right]+\sum_{j}^{N}\left[\begin{array}{cc}
\mathbf{I} & \mathbf{0} \\
\mathbf{0} & \mathbf{M}_{\mathrm{dir}}^{(j)}
\end{array}\right], \quad \mathbf{G}_{\mathrm{tot}}(\omega)=\left[\begin{array}{cc}
\mathbf{0} & -\mathbf{I} \\
\mathbf{K} & \mathbf{C}
\end{array}\right]+\sum_{j}^{N}\left[\begin{array}{cc}
\mathbf{0} & -\mathbf{I} \\
\mathbf{K}_{\mathrm{dir}}^{(j)} & \mathbf{C}_{\mathrm{dir}}^{(j)}
\end{array}\right]
$$

The $\boldsymbol{\Lambda}(\omega, \mathrm{t})$ and $\mathbf{U}(\omega, \mathrm{t})$ variables are the evolutionary spectra terms of $\boldsymbol{\lambda}(t)$ and $\mathbf{u}(t)$ where

$$
\boldsymbol{\lambda}=\left[\begin{array}{c}
\mathbf{q} \\
\dot{\mathbf{q}}
\end{array}\right], \quad \mathbf{u}=\left[\begin{array}{c}
\mathbf{0} \\
\mathbf{f}_{\mathrm{ext}}
\end{array}\right]+\sum_{j}^{N}\left[\begin{array}{c}
\mathbf{0} \\
\mathbf{f}_{\mathrm{rev}}^{(j)}
\end{array}\right]
$$

and $\mathbf{f}_{\text {ext }}$ represents external forcing on the deterministic system.

It can be shown from Eq. (18) that

$$
\boldsymbol{\Lambda}(\omega, t)=\int_{-\infty}^{\infty} \mathbf{H}(\omega, t-\tau) \mathbf{U}(\omega, \tau) \mathrm{e}^{-\mathrm{i} \omega(t-\tau)} \mathrm{d} \tau
$$


where $\mathbf{H}(\omega, t)=\exp \left(-\boldsymbol{\Gamma}_{\text {tot }}^{-1} \mathbf{G}_{\text {tot }}(\omega) t\right) \boldsymbol{\Gamma}_{\text {tot }}^{-1}$ and the limits have been extended to infinity because $\mathbf{H}(\omega, t)$ and $\mathbf{u}(t)$ are zero when $t<0$. Physically, $\mathbf{H}(\omega, t)$ represents the impulse response of the total system (deterministic plus subsystem direct fields) when the mass, damping and stiffness matrices of the direct fields of the subsystems are set to their values found under harmonic excitation at frequency $\omega$. It should be noted that this first order state-space representation is not required if the deterministic system contains a mass term at each degree of freedom.

\subsection{Diffuse field reciprocity relationship}

Equation (23) describes the response in terms of the forcing; however, the statistically modelled subsystems are interested in energies and power flows, which are related to the square of the response and therefore forcing. To combine the deterministic degrees of freedom with the subsystem energies, the diffuse field reciprocity relationship $[16,17]$ shows that under steady excitation conditions the cross-spectrum of the reverberant force generated by a subsystem can be related to the energy in that subsystem. In this subsection, this relationship is investigated for the transient case and modified to comply with the Priestley notation. If $\mathbf{u}_{\mathrm{rev}}^{(j)}(t)$ and $\mathbf{U}_{\mathrm{rev}}^{(j)}(\omega, t)$ are respectively the time varying and evolutionary spectrum terms corresponding to the reverberant force in the jth subsystem, then the mean square correlation matrix of the reverberant force terms can be investigated using the evolutionary spectrum terms since

$$
\mathbf{S}_{\mathrm{u}, \mathrm{rev}}^{(j)}(\mathrm{t})=\mathrm{E}\left[\mathbf{u}_{\mathrm{rev}}^{(j)}(t) \mathbf{u}_{\mathrm{rev}}^{(j)}(t)^{\mathrm{T}}\right]=\int_{0}^{\infty} \mathbf{U}_{\mathrm{rev}}^{(j)}(\omega, t) \mathbf{U}_{\mathrm{rev}}^{(j) * \mathrm{~T}}(\omega, t) \mathrm{d} \omega
$$

By analogy to the steady-state diffuse field reciprocity relationship [17], the evolutionary spectrum terms can be written as

$$
\mathbf{U}_{\mathrm{rev}}^{(j)}(\omega, t) \mathbf{U}_{\mathrm{rev}}^{(j) * \mathrm{~T}}(\omega, t)=d^{(j)}(\omega, t) \frac{2 \alpha E_{j}(\omega, t)}{\pi n_{j}}\left[\begin{array}{cc}
\mathbf{0} & \mathbf{0} \\
\mathbf{0} & \mathbf{C}_{\mathrm{dir}}^{(j)}(\omega)
\end{array}\right]
$$

where $E_{j}(\omega, t)$ is the evolutionary spectrum of the energy in the jth subsystem, the conventional $\operatorname{Im}\left[\mathbf{D}_{\mathrm{dir}}^{(j)}(\omega)\right] / \omega$ term [17] has been replaced by $\mathbf{C}_{\mathrm{dir}}^{(j)}(\omega), \alpha$ depends on the number of connections to the subsystem and whether energy is lost or gained through the connection [17] and $n_{j}$ is the modal density of the $j$ th subsystem. The function $d^{(j)}(\omega, t)$ is included to account for the transient build-up of a diffuse field within a subsystem after an impulse has been applied. It should be noted that Eq. (25) is a factor of two smaller than the steady-state version because the value of interest is the ensemble average of time variables in Eq. (24) rather than frequency variables as in the steady-state approach and is thus half the size. 
The term $\mathbf{U}_{\text {rev }}^{(j)}(\omega, t) \mathbf{U}_{\text {rev }}^{(j) * \mathrm{~T}}(\omega, t)$ in Eq. (25), when examined at a given frequency, contains a scalar time-varying term multiplying a matrix and as such Eq. (25) can be rewritten for the purposes of later use as

$$
\mathbf{U}_{\mathrm{rev}}^{(j)}(\omega, t) \mathbf{U}_{\mathrm{rev}}^{(j) * \mathrm{~T}}(\omega, t)=a^{(j)}(\omega, t)^{2} \overline{\mathbf{U}}_{\mathrm{rev}}^{(j)}(\omega) \overline{\mathbf{U}}_{\mathrm{rev}}^{(j) * \mathrm{~T}}(\omega)
$$

where

$$
a^{(j)}(\omega, t)=\sqrt{d^{(j)}(\omega, t) \frac{2 \alpha E_{j}(\omega, t)}{\pi n_{j}}}, \quad \overline{\mathbf{U}}_{\mathrm{rev}}^{(j)}(\omega) \overline{\mathbf{U}}_{\mathrm{rev}}^{(j) * \mathrm{~T}}(\omega)=\left[\begin{array}{cc}
\mathbf{0} & \mathbf{0} \\
\mathbf{0} & \mathbf{C}_{\mathrm{dir}}^{(j)}(\omega)
\end{array}\right]
$$

The derivation of the diffuse field reciprocity relationship under steady conditions $[16,17]$ assumes a diffuse field is present in the subsystem. However, under impulsive loading, this is not the case and the response of a subsystem immediately after an impulse will be largely governed by the deterministic direct field. As time progresses, over the ensemble of random systems, the response is scattered and eventually becomes diffuse. The reverberant forces will therefore show a largely deterministic character at low times, possibly with a time delay as waves travel from the impulse to the location of the connection to the deterministic system, and a diffuse character at late times at which Eqs. (26-28) with $d^{(j)}(\omega, t)=1$ will accurately model the response. The term $d^{(j)}(\omega, t)$ can be selected to mitigate inaccuracies due to this effect and is investigated further numerically in Section 4.1. The accuracy of the reverberant force calculation will depend on the relative contributions of the direct and reverberant fields, although in SEA applications it is assumed that the reverberant field dominates and so the transient response is not expected to contain inaccuracies any larger than those found in steady-state SEA or hybrid FE-SEA models that neglect the direct field.

A similar issue has been discussed for TSEA where instantaneous transfer of energy between subsystems is predicted even before waves from the source have had time to travel to a connection to another subsystem. It has been suggested $[10,11,19]$ that solving the TSEA differential equation numerically with a time-step related to the time taken for energy to travel through a subsystem can account for this issue, although [13] suggests that with appropriate SEA conditions of low damping and weak coupling, the problem should not be significant. Viewing the issue from the perspective of the transient hybrid method sheds further light on the situation, where since Eq. (26) will be directly related to the hybrid equivalent of the SEA coupling loss factors, one method for accounting for the build-up of a reverberant force with time in TSEA would be to modulate the coupling loss factors with a time-varying term similar to $d^{(j)}(\omega, t)$. Whilst this should not be necessary for most SEA applications due to weak coupling, it may be more applicable to hybrid FE-SEA systems because coupling to the deterministic system could be stronger. 


\subsection{Power balance}

The equations of motion and diffuse field reciprocity relationship of the previous section can now be assembled to explore power flow through the system. By investigating the power transfer through each subsystem, a matrix equation similar to the transient SEA equation [13] is produced

$$
\dot{\mathbf{E}}(\omega, t)+\mathbf{A}_{\eta}(\omega) \mathbf{E}(\omega, t)=\mathbf{p}(\omega, t)+\mathbf{p}_{\mathrm{ext}}(\omega, t)
$$

where $\mathbf{E}(\omega, t)$ is a vector of the evolutionary spectra of the energy in each subsystem and $\mathbf{A}_{\eta}$ is a diagonal matrix accounting for internal losses in a subsystem such that $A_{\eta, j k}=\delta_{j k} \omega \eta_{j}$ with $\eta_{j}$ being the loss factor of the $j$ th subsystem. The vectors $\mathbf{p}(\omega, t)$ and $\mathbf{p}_{\text {ext }}(\omega, t)$ represent the power into each subsystem from the deterministic system and external forcing applied directly to the subsystems respectively. The power, $p_{j}(t)$, absorbed by the $j$ th subsystem can be found by splitting Eq. (16) into its direct field and reverberant force components and pre-multiplying by $\dot{\mathbf{q}}^{\mathrm{T}}$, to yield

$$
p_{j}(t)=\mathrm{E}\left[\dot{\mathbf{q}}^{\mathrm{T}} \mathbf{f}^{(j)}\right]=\mathrm{E}\left[\dot{\mathbf{q}}^{\mathrm{T}} \mathbf{C}_{\text {dir }}^{(j)} \dot{\mathbf{q}}-\dot{\mathbf{q}}^{\mathrm{T}} \mathbf{f}_{\text {rev }}^{(j)}\right]
$$

where the reactive power absorbed by the direct field has been ignored. By noting that Eq. (30) can be converted into the state-space variables and the relationship between mean square correlation matrix and evolutionary spectra in Eq. (24), the evolutionary spectrum of the power absorbed by the $j$ th subsystem, $p_{j}(\omega, t)$, can be calculated as

$$
p_{j}(\omega, t)=\sum_{m n}\left[\begin{array}{ll}
\mathbf{0} & \mathbf{0} \\
\mathbf{0} & \mathbf{C}_{\mathrm{dir}}^{(j)}(\omega)
\end{array}\right]_{m n}\left\{\boldsymbol{\Lambda}(\omega, t) \boldsymbol{\Lambda}^{* \mathrm{~T}}(\omega, t)\right\}_{m n}-\operatorname{Re}\left[\sum_{m}\left\{\boldsymbol{\Lambda}(\omega, t) \mathbf{U}_{\mathrm{rev}}^{(j) * \mathrm{~T}}(\omega, t)\right\}_{m m}\right]
$$

The evolutionary spectrum of the mean square response, $\boldsymbol{\Lambda}(\omega, t) \boldsymbol{\Lambda}^{* \mathrm{~T}}(\omega, t)$, can be calculated using Eq. (23) and converting $\mathbf{U}(\omega, t)$ into its constituents $\mathbf{U}_{\text {ext }}(\omega, t)+\sum_{j}^{N} \mathbf{U}_{\mathrm{rev}}^{(j)}(\omega, t)$ where $\mathbf{U}_{\text {ext }}(\omega, t)$ is the evolutionary spectrum of any external input excitation on the deterministic system. Noting that the reverberant forces from the subsystems are uncorrelated to each other and to any external input force and using Eq. (26) the evolutionary spectrum of mean square response becomes

$$
\begin{aligned}
& \boldsymbol{\Lambda}(\omega, t) \boldsymbol{\Lambda}^{* \mathrm{~T}}(\omega, t)=\sum_{j}^{N} \int_{-\infty}^{\infty} \mathbf{H}(\omega, t-\tau) a^{(j)}(\omega, \tau) \mathrm{e}^{-\mathrm{i} \omega(t-\tau)} \mathrm{d} \tau \overline{\mathbf{U}}_{\mathrm{rev}}^{(j)}(\omega) \overline{\mathbf{U}}_{\mathrm{rev}}^{(j) * \mathrm{~T}}(\omega) \times \\
&\left\{\int_{-\infty}^{\infty} \mathbf{H}(\omega, t-\tau) a^{(j)}(\omega, \tau) \mathrm{e}^{-\mathrm{i} \omega(t-\tau)} \mathrm{d} \tau\right\}^{* \mathrm{~T}}+ \\
& \int_{-\infty}^{\infty} \mathbf{H}(\omega, t-\tau) \mathbf{U}_{\text {ext }}(\omega, \tau) \mathrm{e}^{-\mathrm{i} \omega(t-\tau)} \mathrm{d} \tau \times \\
&\left\{\int_{-\infty}^{\infty} \mathbf{H}(\omega, t-\tau) \mathbf{U}_{\text {ext }}(\omega, \tau) \mathrm{e}^{-\mathrm{i} \omega(t-\tau)} \mathrm{d} \tau\right\}^{* \mathrm{~T}} .
\end{aligned}
$$


Equations (27) and (28) can then be substituted into Eq. (32) to relate the mean square response to the energy of each subsystem. Using a similar approach, the evolutionary cross-spectrum in the rightmost term in Eq. (31) can be calculated as

$$
\boldsymbol{\Lambda}(\omega, t) \mathbf{U}_{\mathrm{rev}}^{(j) * \mathrm{~T}}(\omega, t)=a^{(j)}(\omega, t) \int_{-\infty}^{\infty} \mathbf{H}(\omega, t-\tau) a^{(j)}(\omega, \tau) \mathrm{e}^{-i \omega(t-\tau)} \mathrm{d} \tau \overline{\mathbf{U}}_{\mathrm{rev}}^{(j)}(\omega) \overline{\mathbf{U}}_{\mathrm{rev}}^{(j) * \mathrm{~T}}(\omega)
$$

By combining Eqs. (31-33) the power into each subsystem at a given time and frequency can be calculated. This can be substituted into Eq. (29) to generate the time derivative of the energy in each subsystem. The equations can then be time-integrated numerically at a fixed frequency to generate both the time evolution of the energy in each SEA subsystem and the mean square correlation matrix of the response of the deterministic degrees of freedom. It can be noted that under steady excitation conditions, the equations collapse to the standard steady-state equations of Shorter and Langley [4] as shown in the appendix.

\subsection{Impulsive excitation}

If an impulse on the system acts on an SEA subsystem, then it can be applied as an initial condition on the energy of the subsystem using the formula given in [13]. If, however, the impulse is applied to the deterministic system, a more involved procedure is required. In this case, initially after the impulse, the response of the deterministic system will exhibit largely deterministic behaviour that does not fit into the Priestley formulation adopted above. However, provided a reasonable approximation of the evolutionary spectrum of the power input into the subsystems from the excited deterministic system can be made, the method should provide accurate results.

The average response of the deterministic system due to the impulse and not including reverberant forces can be calculated from the impulse response of Eq. (18) and denoted $\mathbf{q}_{\text {imp }}(t)$. The power absorbed by the jth subsystem due to the impulse response of the deterministic system, denoted $p_{\text {imp }}^{(j)}(t)$, can then be calculated using Eq. (30) where the right-hand term is zero because the reverberant force is uncorrelated to the motion. However, the variable, $\mathbf{q}_{\text {imp }}(t)$, here is a deterministic function of time therefore does not take the assumed Priestley form of Eq. (8) and so the evolutionary spectrum of the power on the left-hand side of Eq. (31) does not follow automatically.

An evolutionary spectrum formulation will be representative provided both the time and frequency content of the response are captured. A reasonable approximation of the time response can be found by taking a smoothed version of $p_{\mathrm{imp}}^{(j)}(t)$, denoted $\bar{p}_{\mathrm{imp}}^{(j)}(t)$, which can be achieved using a moving mean filter. The frequency profile introduced into a subsystem from the impulse can be best estimated from the Fourier transform of the impulse response and is therefore calculated as the frequency 
response that would be observed under steady-state band-limited loading. This can be calculated by combining the first term on the right-hand side of Eq. (31) with the terms involving external forcing in Eq. (32) where the forcing is band-limited to the frequencies of the impulse and is constant with time. This is equivalent to the steady-state approach of Eq. (14) in [4] which uses dynamic stiffness matrices. Denoting the frequency content of the power input to the $j$ th subsystem as $P_{\mathrm{imp}}^{(j)}(\omega)$, the evolutionary spectrum of the power absorbed from the impulse can then be approximated as

$$
p_{\mathrm{imp}}^{(j)}(\omega, t)=\bar{p}_{\mathrm{imp}}^{(j)}(t) \frac{P_{\mathrm{imp}}^{(j)}(\omega)}{\int_{\omega_{l}}^{\omega_{u}} P_{\mathrm{imp}}^{(j)}(\omega) \mathrm{d} \omega}
$$

where the normalisation has been applied such that $\int_{\omega_{l}}^{\omega_{u}} p_{\mathrm{imp}}^{(j)}(\omega, t) \mathrm{d} \omega=\bar{p}_{\mathrm{imp}}^{(j)}(t)$. This is sufficient to calculate the evolutionary spectra of the energy in the subsystems and when the mean square correlation matrix of the deterministic response due to the subsystem energies and any external loads other than the impulse is calculated using Eq. (34), the mean square values from $\mathbf{q}_{\text {imp }}(t)$ must be added to generate the total response.

\section{Numerical validation}

The preceding theory is validated numerically in this section by comparing transient hybrid FE-SEA results to finite element (FE) results of systems containing plates and beams. The Lagrange-RayleighRitz method and commercial FE package COMSOL are used to build an ensemble containing 500 realisations of FE models in which the SEA components are randomised by adding 10 masses each of $1 \%$ of the subsystem mass at random locations.

\subsection{Diffuse field reciprocity relationship}

In this subsection, the transient diffuse field reciprocity relationship discussed in Section 3.2 is investigated via an impulsively excited single plate system with a single spring to ground as shown in Figure 1. The reverberant force at the spring connection point can be found using the time domain version of Eq. (17) such that

$$
f_{\mathrm{rev}}(t)=8 \sqrt{D \rho h} \dot{x}(t)-k x(t)
$$

where $D, \rho$ and $h$ are the flexural rigidity, density and thickness of the plate respectively and $x(t)$ is the displacement of the plate at the spring connection. The direct field of the plate provides only damping at a single point with damping constant given analytically as $8 \sqrt{D \rho h}$. The plate is aluminium 
with dimensions $0.4 \times 0.7 \times 0.002 \mathrm{~m}$, Young's modulus $71 \mathrm{GPa}$, density $2700 \mathrm{~kg} \mathrm{~m}^{-3}$, Poisson ratio 0.33 and with all edges pinned to allow rotation, but zero displacement. Only out-of-plane modes were considered and an ensemble of 500 realisations was considered with an impulse containing frequencies in the range 1000-2000 Hz. The impulse was applied by taking the inverse Fourier transform of the frequency response over the frequency range of interest.

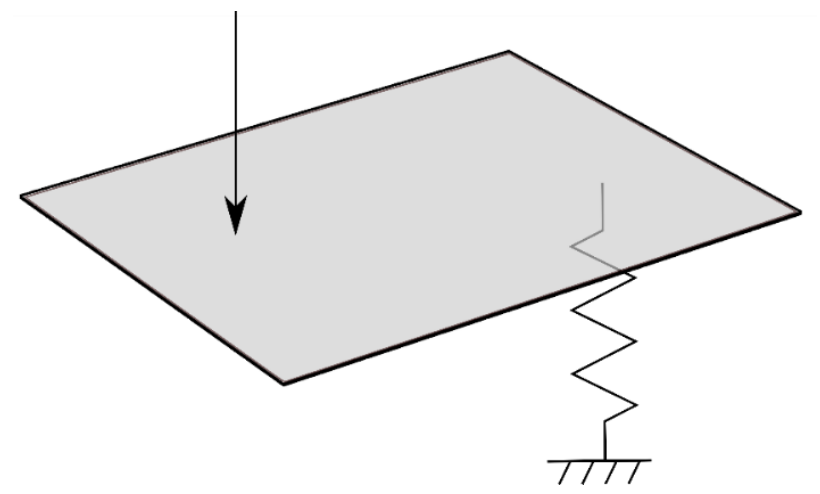

Figure 1 . Single plate system.

Using Eq. (25), the mean square reverberant force can be compared to the total energy of the subsystem to explore the relationship between the two possible forms of the function $d^{(j)}(\omega, t)$. Since the timescale for the build up of the reverberant field is related to the timescale for energy transfer across the plate, denoted $T_{\text {rev }}$, a reasonable estimate of the reverberant force may be given using

$$
d^{(j)}(\omega, t)=1-\exp \left(-\frac{t}{T_{\text {rev }}}\right)
$$

where $T_{\mathrm{rev}}$ can be approximated as the time taken for a wave at the centre frequency of the frequency range of interest to travel a representative length of the plate for example the mean of the length and width. For the case where the plate loss factor, $\eta$, is 0.03 and the spring stiffness is $6 \times 10^{7} \mathrm{~N} \mathrm{~m}^{-1}$, the mean square reverberant force is compared to the prediction from Eq. (24) with $d^{(j)}(\omega, t)=1$ and using Eq. (36) in Figure 2 for both an impulse and steady harmonic input. 


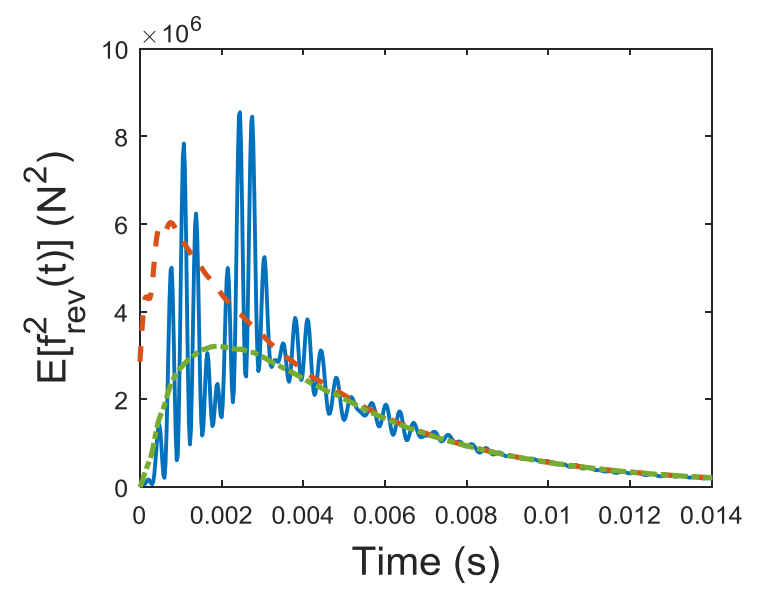

(a)

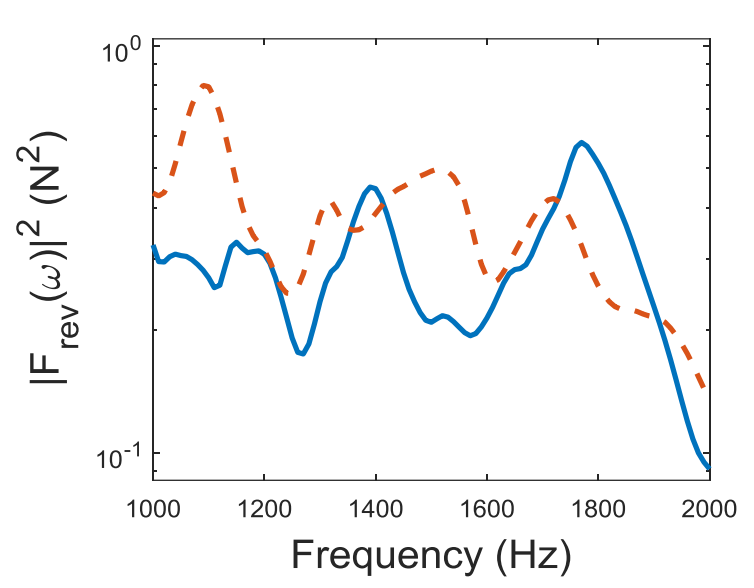

(b)

Figure 2. Mean square reverberant force at the spring connection (a) after an impulse and (b) steady harmonic forcing from simulations (solid) and predicted using Eq. (25) with $d^{(j)}(\omega, t)=1$ (dashed) and Eq. (36) (dash-dot).

A number of differences between the simulated and predicted results are observed. In the time domain there is a short time delay while waves travel out from the impulse to the spring connection point followed by a distinct oscillatory pattern in the reverberant force soon after the impulse. The oscillations then die down and at larger times the prediction from Eq. (25) becomes accurate for both $d^{(j)}(\omega, t)$ functions.

The oscillations after the impulse are a deterministic feature caused by the direct field wavefronts travelling out from the impulse applying a force to the connection point both directly and after reflections from the boundaries. Over the ensemble of random plates, the waves become more scattered with time and so the deterministic features become averaged out. The response from the direct field is investigated in Figure 3 where the spring stiffness is reduced to zero meaning that the reverberant force depends only on the plate velocity at the point. The velocity at a point on an infinite plate from an impulse is known analytically and so the reverberant force from the direct field can be calculated and compared to the response from the ensemble of randomised plates. A number of reflections from the plate boundary have also been included by assuming reflection off the pinned edges simply inverts the sign of the incoming wave, and the resulting reverberant force prediction is seen to match the oscillations of the simulated results reasonably well until the field becomes diffuse. A more accurate representation of the reverberant forces could be devised using a method such as this to include the direct field response, although this is not explored further in this paper. 


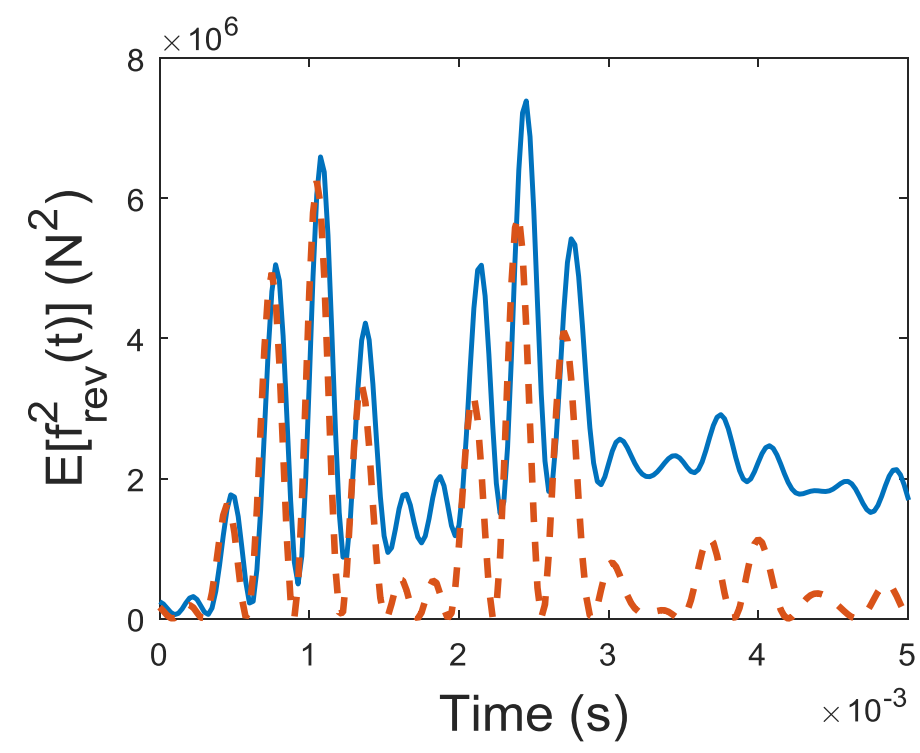

Figure 3. Mean square reverberant force at the spring connection with zero spring force after an impulse. Response from simulations (solid) and predicted from only the direct field with reflections (dashed).

The interaction between the direct and reverberant field is more apparent under impulsive loading, although the two also co-exist under steady loading. In general, steady-state SEA-based methods assume subsystems have low damping and so the reverberant field is considerably larger than the direct field which is therefore neglected. Under impulsive loading with lower damping, the time over which the reverberant field acts would dominate over the early times over which the direct field acts and so by a similar argument, the direct field responses could be neglected. Under these conditions, prediction of the reverberant force using $d^{(j)}(\omega, t)=1$ is expected to be reasonable and the steadystate and transient methods are expected to yield results of comparable accuracy. By using a higher fidelity form of the function $d^{(j)}(\omega, t)$ the range of applicability of the method may be increased, although the exact form of the function will depend on each individual circumstance and will inevitably complicate the method.

\subsection{Plate and beam system}

The system illustrated in Figure 4 and based on that used to validate the steady-state FE-SEA theory in [4] is investigated by comparing the transient hybrid FE-SEA method of Section 3 with FE simulations. The system consists of two plates coupled via point connections to a beam where the flexural wavefields of the plates are modelled using SEA and the beam is modelled using FE. The point connections are constrained to move vertically such that in-plane deformation of the plates is not generated by the flexure of the beam thus only flexural waves need to be considered in the plates. The plates and beam have the same properties as the system in Section 4.1 with plate dimensions 
$0.28 \times 0.35 \times 0.001 \mathrm{~m}$ and $0.3 \times 0.35 \times 0.00075 \mathrm{~m}$, loss factor 0.01 and further details are documented in detail in [4].

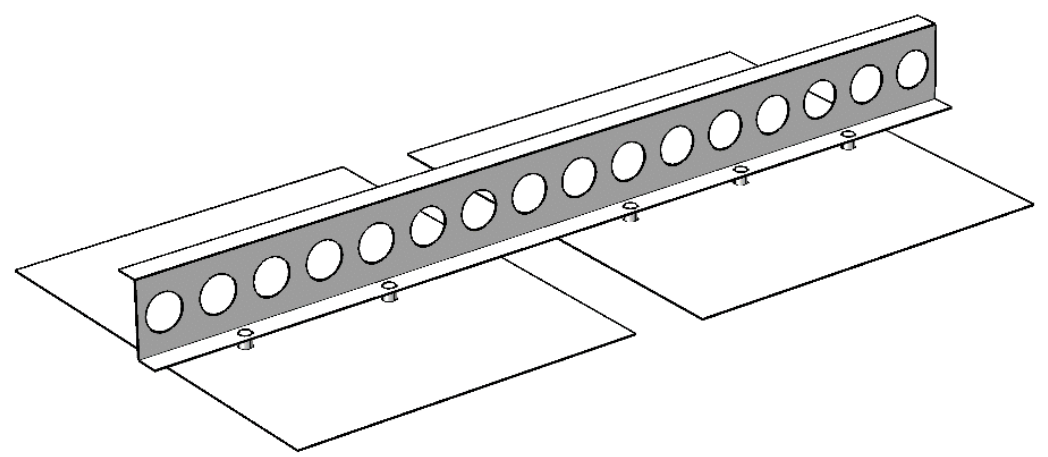

Figure 4. Plate and beam system.

Both the benchmark simulations where the entire system is modelled using FE and the hybrid analysis where only the deterministic beam is modelled with FE contain a large number of nodes and therefore degrees of freedom. In order to reduce the number of degrees of freedom, a modal decomposition was performed and the modal rather than nodal degrees of freedom were used in Eq. (12) and the subsequent analysis. For the benchmark simulations, the modes of the unrandomised system were calculated and the system was randomised to create an ensemble by projecting additional random masses on the plates onto the modal basis functions. The same method is used in [4] where more details can be found. The frequency range of interest is $1500-2500 \mathrm{~Hz}$ below which the entire system contains approximately 171 modes and so conservatively, the first 300 modes were selected for the analysis. Similarly, the isolated beam system used as the FE part of the hybrid analysis contains 19 modes below $2500 \mathrm{~Hz}$ and so 25 modes were selected for the hybrid analysis which was also performed in modal coordinates.

An impulse containing frequencies in the range $1500-2500 \mathrm{~Hz}$ is applied to one of the plates and the energy of the plates and the velocity response of a point approximately in the middle and at the top of the beam are displayed in Figure 5 from a full FE model and hybrid FE-SEA model. The hybrid method has been evaluated at 50 frequencies and results are shown both when $d^{(j)}(\omega, t)$ is set to unity and when it is set using Eq, (36). The initial energy in the excited plate is calculated using the method of [13] which provides an overprediction of approximately $50 \%$ most likely due to the effect of the beam on the plate, although the decay is well predicted. Overall the predictions are reasonably close to the average simulated values with a relatively small difference between the results from using the different $d^{(j)}(\omega, t)$ functions. A slightly lower and slower response is predicted using Eq. (36) due 
to its time lag, although in this example using $d^{(j)}(\omega, t)=1$ provides slightly improved results both in terms of rise times and peak values.

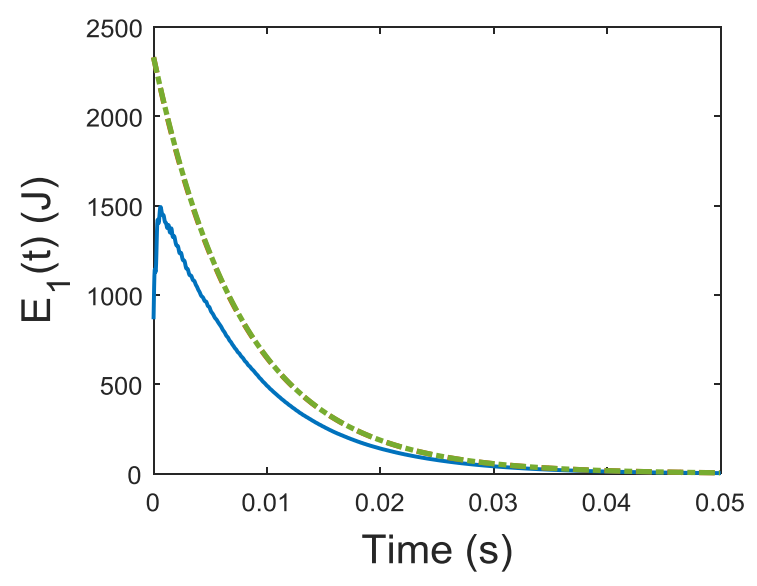

(a)

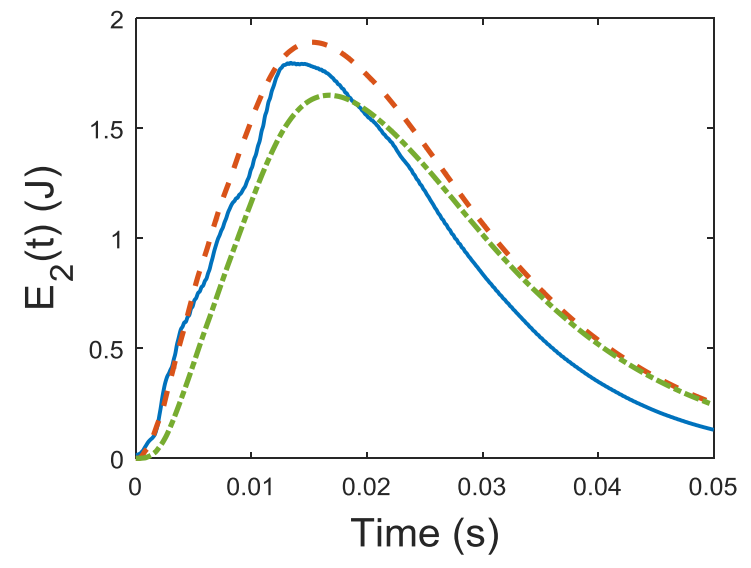

(b)

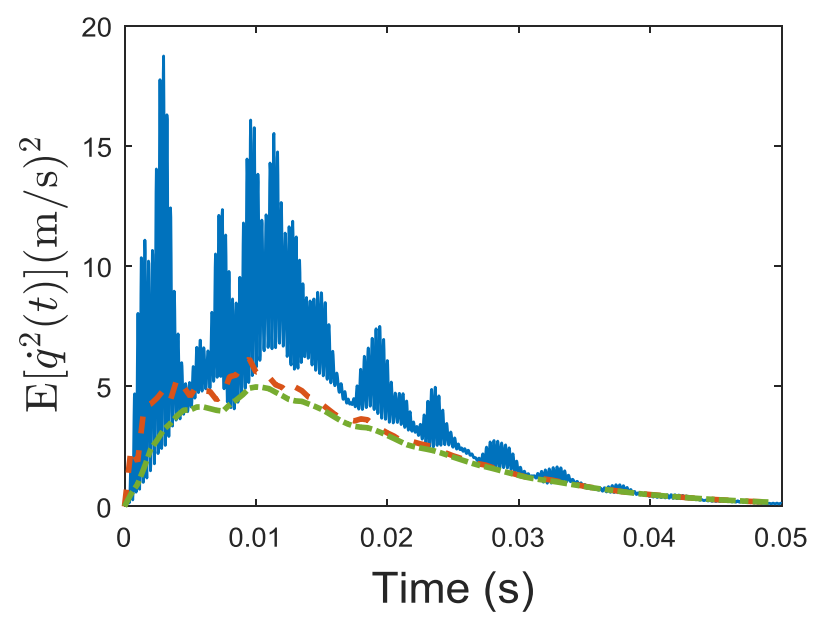

(c)

Figure 5. System response when an impulse is applied to a plate from simulations (solid) and transient hybrid method with $d^{(j)}(\omega, t)=1$ (dashed) and using Eq. (36) (dash-dot). (a) Plate one energy, (b) plate two energy and (c) mean square velocity of point on the beam.

The mean square response of the observation point on the beam exhibits strong deterministic oscillations at low times due to the deterministic effects of the direct field discussed in Section 4.1. If these results are time-averaged to remove the oscillations, the hybrid method would provide a reasonable prediction, with at worst an approximately 50\% underprediction. When the oscillations are included, at their peak they are approximately six times larger than the hybrid prediction. The 
forces acting on the deterministic system could potentially be modelled with an improved model of the reverberant field build up including the direct field in more detail, but as it stands it is a deficiency in the method as it cannot exhibit these deterministic effects.

Since SEA-based methods are approximate, they are often considered reasonable if they predict the response within $3 \mathrm{~dB}$ or approximately a factor of two. The general response of the system is predicted to within $3 \mathrm{~dB}$ and so the method can be considered comparable to other SEA-based methods. Additionally, steady-state hybrid FE-SEA results are most commonly plotted on a logarithmic scale which can, due to their range, hide errors that appear obvious on a linear scale such as those presented here.

The evolutionary spectra of the plate energies and mean square velocity of a point on the beam from the transient hybrid FE-SEA method using $d^{(j)}(\omega, t)=1$ are presented in Figure 6 . These cannot be directly compared with FE simulations as no such spectrum is calculated and so are presented to illustrate the time and frequency content in the response. It is clearly seen that the broad frequency content of the impulsively excited plate is filtered through the beam natural frequencies such that only frequencies around the beam resonances are passed through to the second plate. The relative magnitudes of the frequencies found at the point on the beam and the plate differ because a mode that passes energy between the plates easily may not exhibit a significant response at the observation point on the beam and vice versa. 


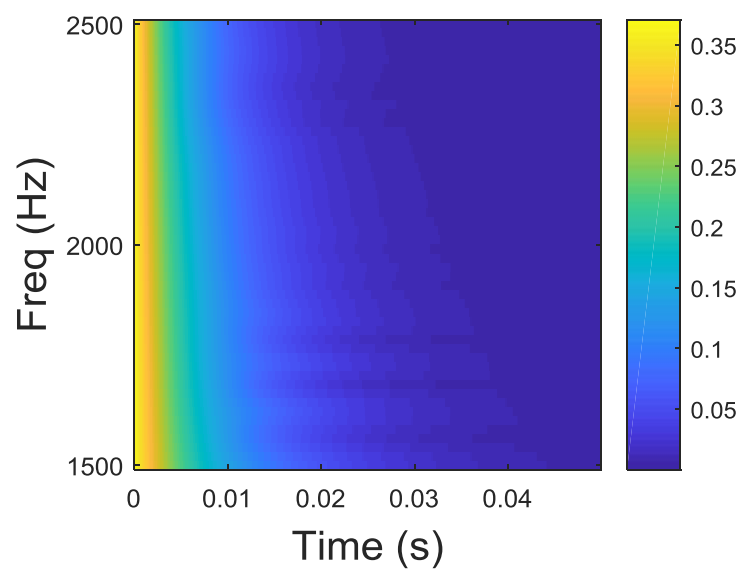

(a)

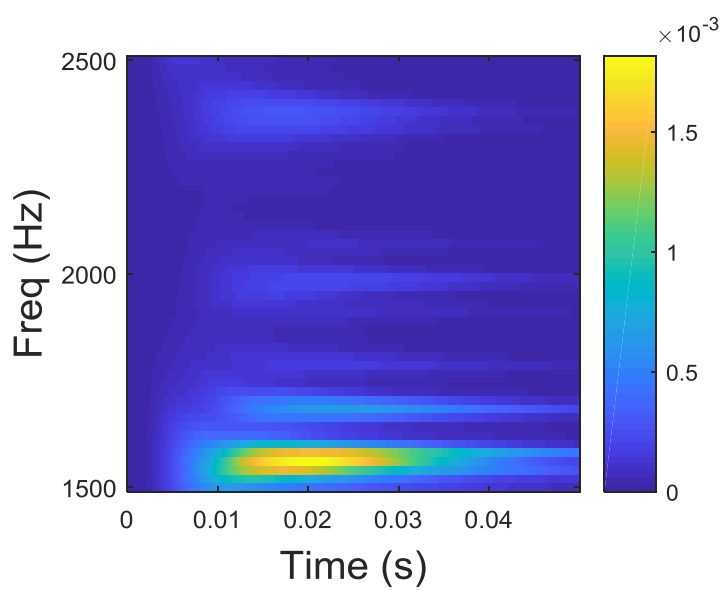

(b)

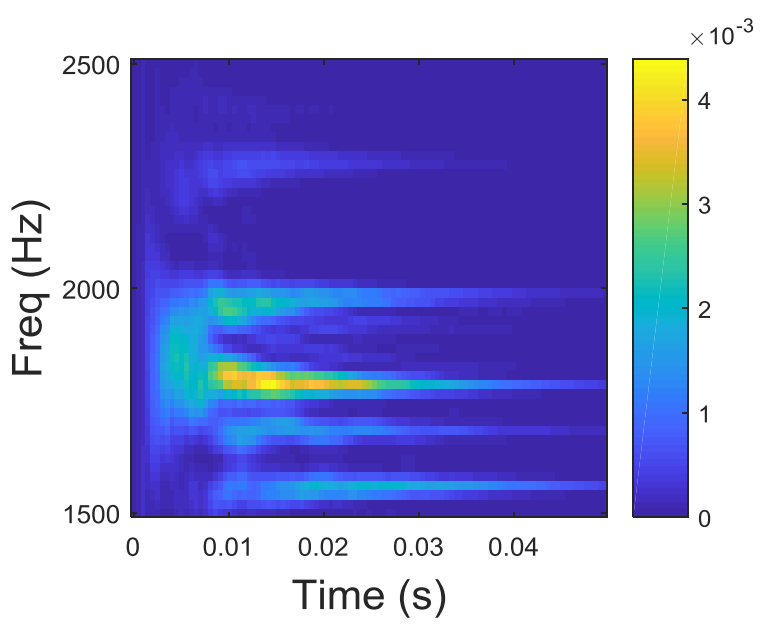

(c)

Figure 6. Evolutionary spectra from the transient hybrid FE-SEA method of (a) plate one energy, (b) plate two energy and (c) mean square velocity of a point on the beam when an impulse is applied to a plate.

Alternatively, the impulse could be applied to the beam, in which case the beam would initially oscillate in a relatively deterministic manner and inject energy into the plates. Figure 7 displays this case where the impulse is applied near one end of the beam and the beam observation point response has been smoothed using a moving average filter to more easily compare it to the transient hybrid method. Here the beam velocity predicted by the hybrid method matches the benchmark very closely because it largely consists of the deterministic impulse response of the beam with the plates applying effective damping which the hybrid theory picks up very well. The energy transfer into the plates is also well accounted for, with both the rise and decay times and peak energies matching the FE simulations closely giving approximately 16 and $22 \%$ underpredictions of the peaks. Here the form of 
function $d^{(j)}(\omega, t)$ is less significant since energy builds up slowly within the subsystems and is therefore set to unity.

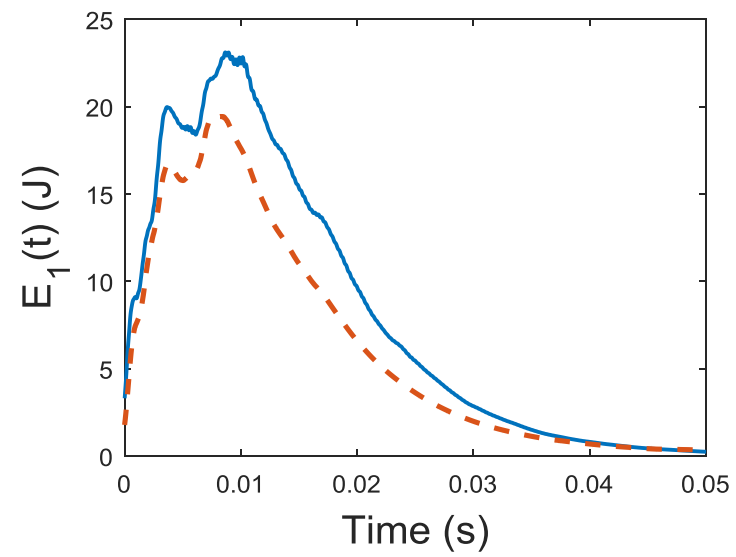

(a)

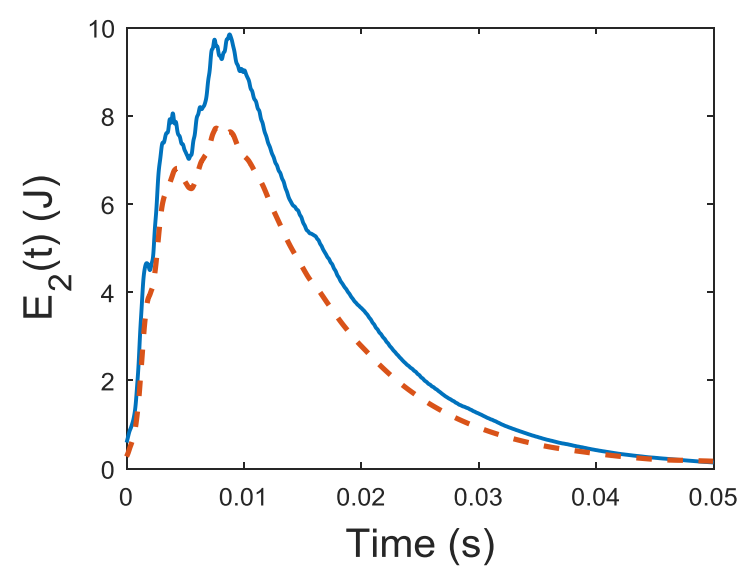

(b)

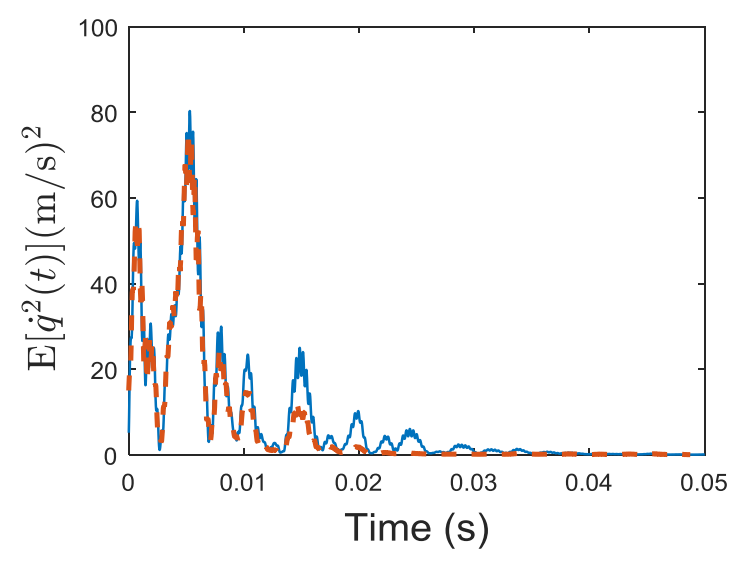

(c)

Figure 7. System response when an impulse is applied to the beam from simulations (solid) and transient hybrid method (dashed). (a) Plate one energy, (b) plate two energy and (c) mean square velocity of point on the beam.

The evolutionary spectra of the responses are displayed in Figure 8 where the dominant frequencies of the beam that are excited by the impulse are transmitted through to the plates. As before, different frequencies dominate in each plate because each beam mode shape may transmit energy more favourably into one plate than the other. The evolutionary spectra of the beam observation point predominantly consists of the deterministic response which is converted into evolutionary spectrum form by combining the time and frequency responses. The structure of the spectrum is therefore naturally an approximation, although the response in the time or frequency domain is accurate. 


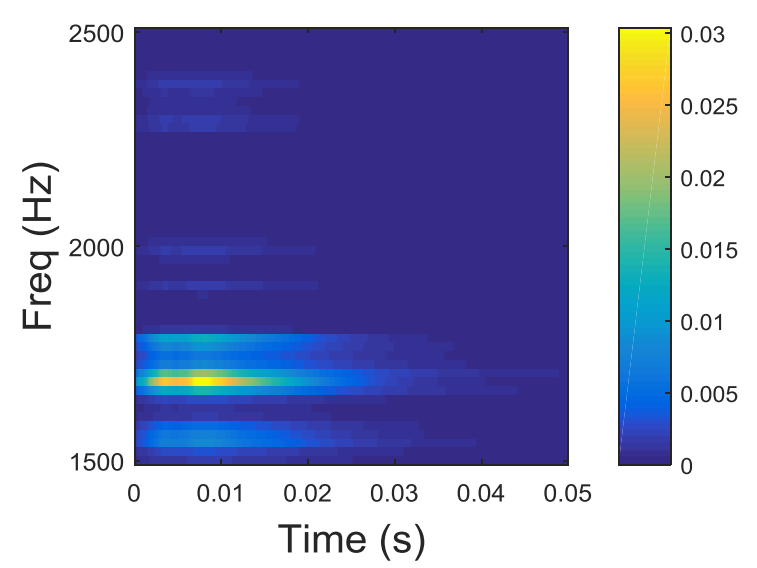

(a)

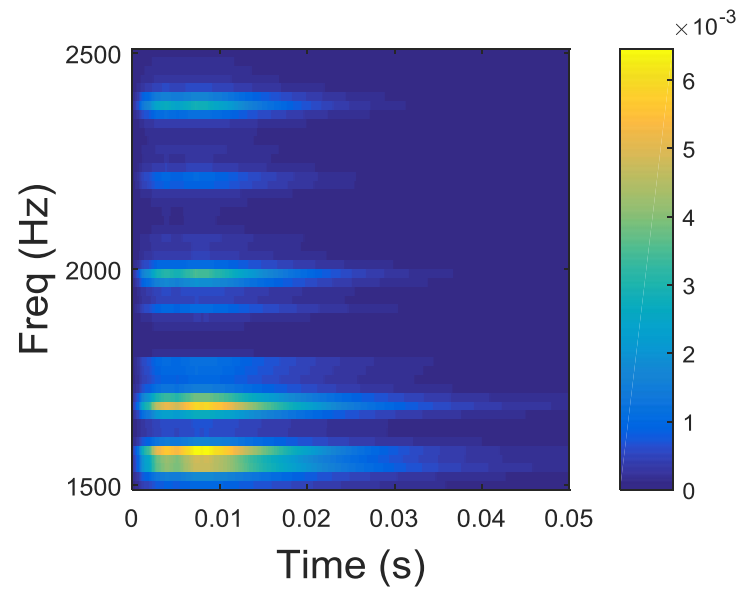

(b)

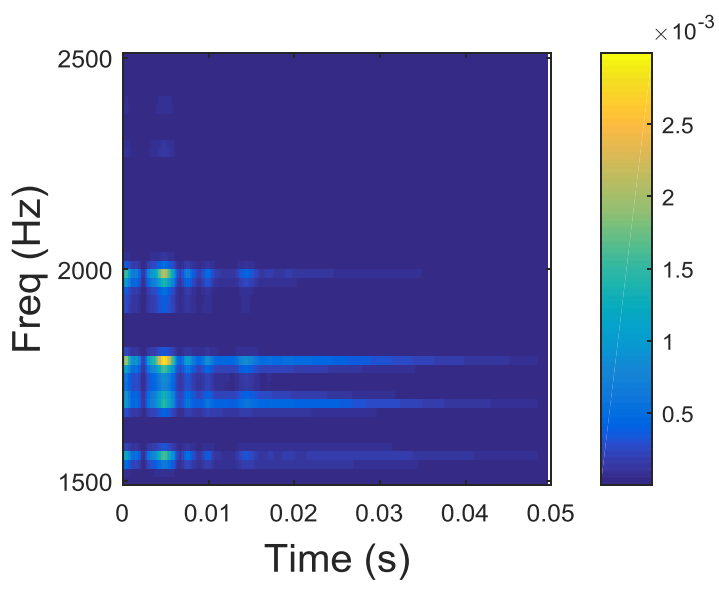

(c)

Figure 8. Evolutionary spectra from the transient hybrid FE-SEA method of (a) plate one energy, (b) plate two energy and (c) mean square velocity of a point on the beam when an impulse is applied to the beam.

The transient hybrid FE-SEA method of Section 3 can also be applied when the loading is steady (or slowly time-varying), but the system has not reached dynamic equilibrium. Figure 9 displays the system response for the case where a steady band-limited random load is applied at a point on a plate when the system is initially at rest. The load is applied as white noise that only acts on the modes within the frequency band and for the FE simulations the equations of motion are reformulated into the non-stationary Lyapunov equation solved with numerical integration. The constant lines on the figures represent the responses calculated using steady-state theory and the transient responses are found to asymptote towards them showing that the transient hybrid method reduces to the steadystate one. There are slight errors in the final values due to the calculation methods used. The form of the time responses predicted by the hybrid method match the simulations closely, although the final 
value can be inaccurate due to the approximations in the steady-state method. This highlights that the transient method can be expected to yield results of a similar accuracy as the steady-state method. The times taken for the hybrid and benchmark simulations were $900 \mathrm{~s}$ and $1.6 \times 10^{4} \mathrm{~s}$ respectively meaning that for this case, the hybrid method provides an 18 times speed improvement. The simulations were performed on a single core and it is expected that the hybrid method could be sped up significantly with optimisation.

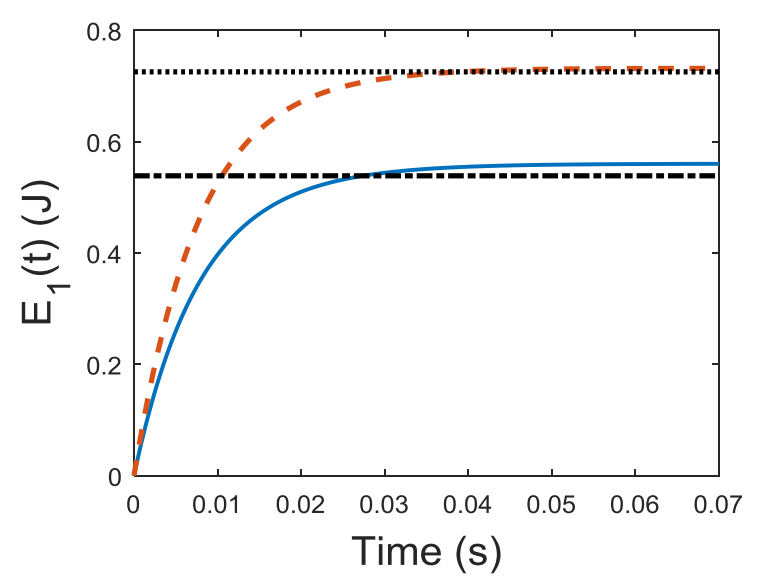

(a)

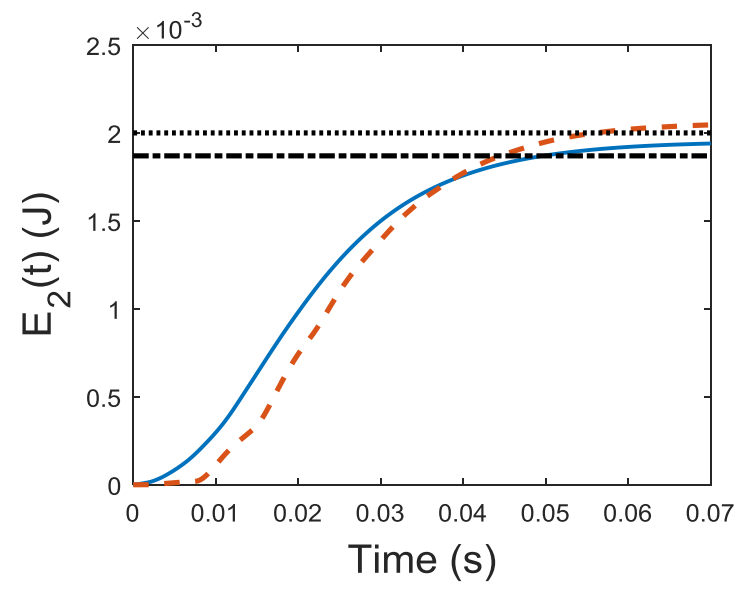

(b)

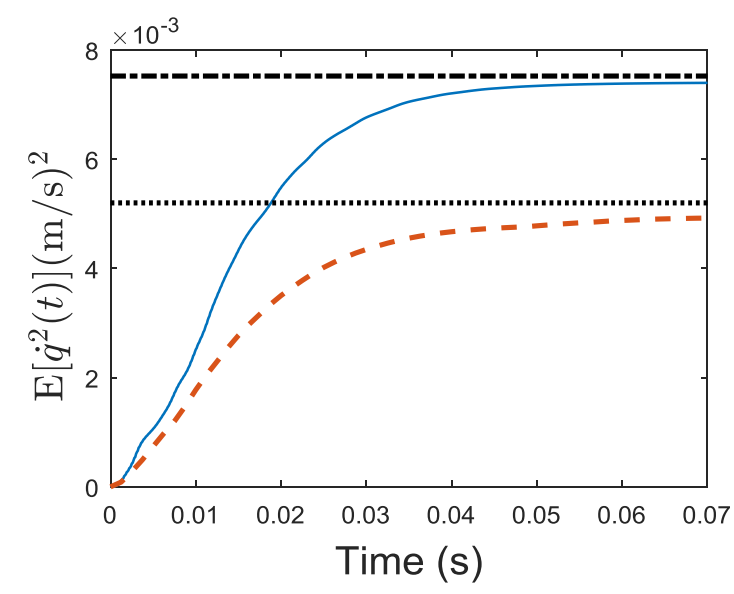

(c)

Figure 9. System response when a steady load is applied to one plate and the system is initially at rest from simulations (solid) and transient hybrid method (dashed). Dash-dot and dotted lines represent response with steady-state excitation from simulations and the hybrid method of [4] respectively. (a) Plate one energy, (b) plate two energy and (c) mean square velocity of point on the beam. 
The evolutionary spectra from the transient hybrid method are displayed in Figure 10. At large times, the spectra are stationary and represent the steady-state frequency responses with peaks at the natural frequencies of the beam as displayed in Figure 10(d) which compares the steady-state results in plate two with the evolutionary spectrum at a large time.

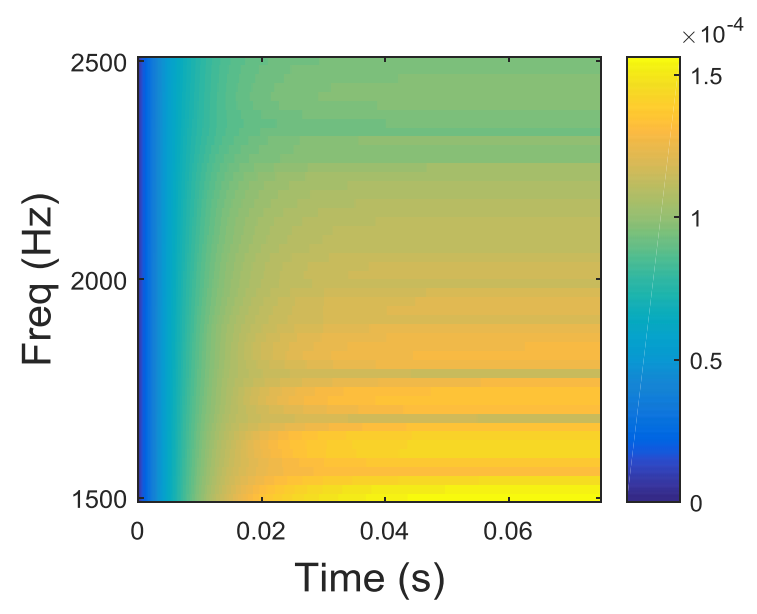

(a)

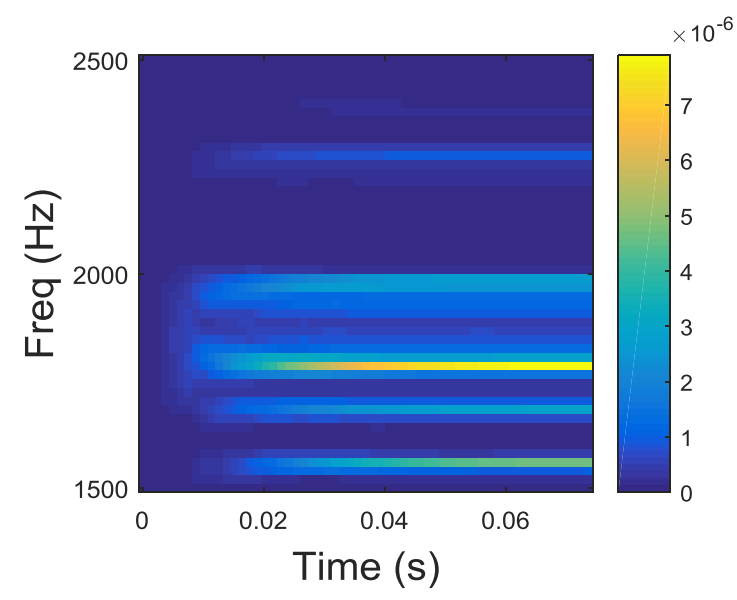

(c)

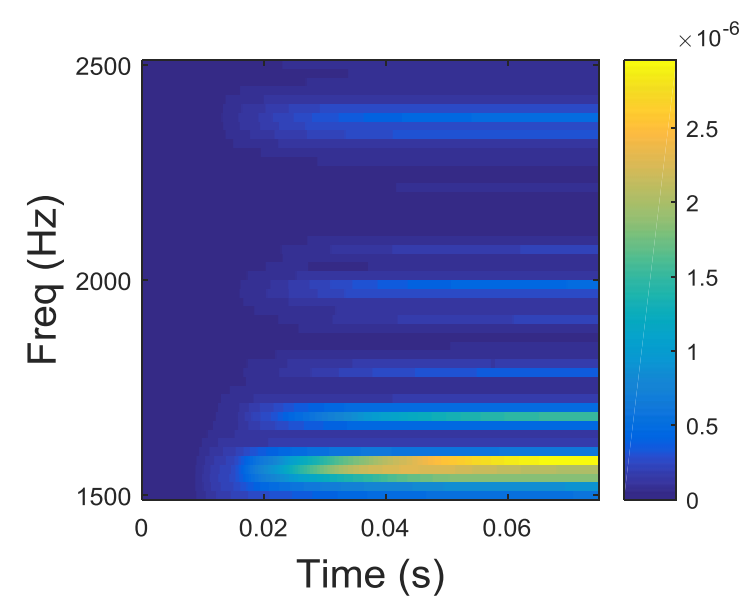

(b)

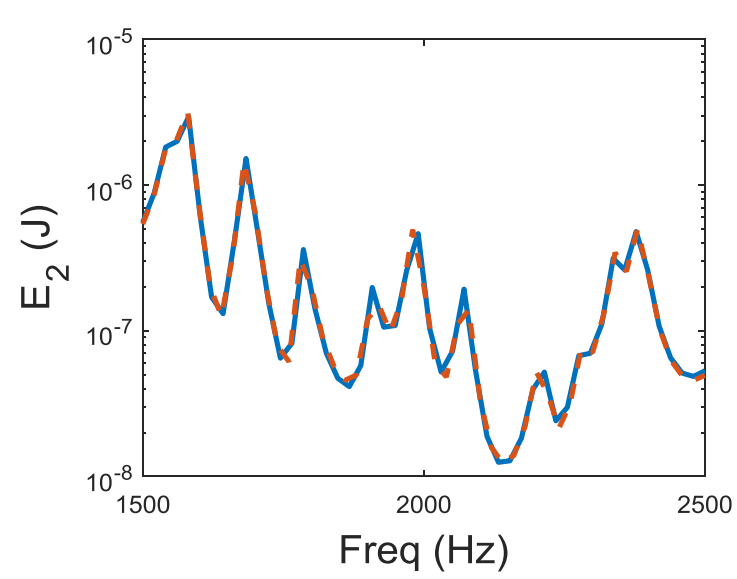

(d)

Figure 10. Evolutionary spectra from the transient hybrid FE-SEA method of (a) plate one energy, (b) plate two energy and (c) mean square velocity of a point on the beam when a steady load is applied to one plate and the system is initially at rest. (d) Frequency content of energy in plate two from transient hybrid (solid) and steady-state hybrid (dashed) methods.

Figures 11 and 12 display the time responses and evolutionary spectra when steady loading is applied to a point on the beam and the system is initially at rest. As with excitation on the SEA subsystem, the results asymptote towards the steady-state results, although the steady value from the two methods does differ. The form of the time histories from both the transient hybrid method and simulations 
again agrees well, particularly for the mean square beam velocity which is predominantly determined by deterministic effects that are modelled accurately by the FE beam model.

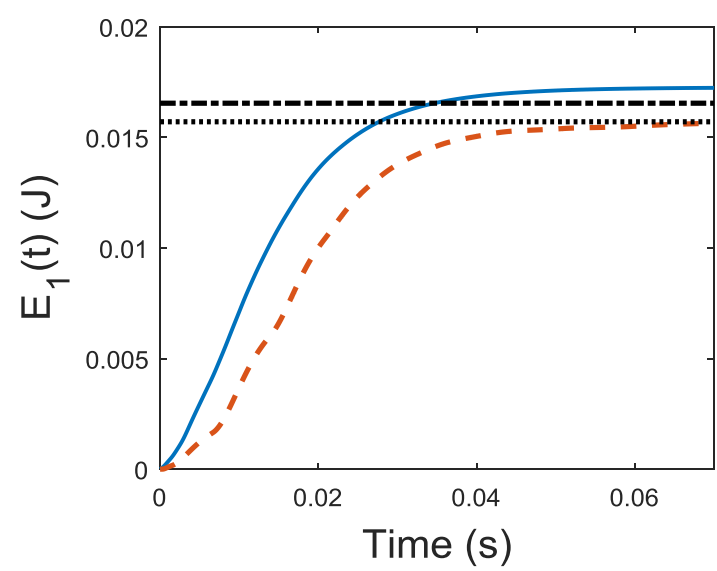

(a)

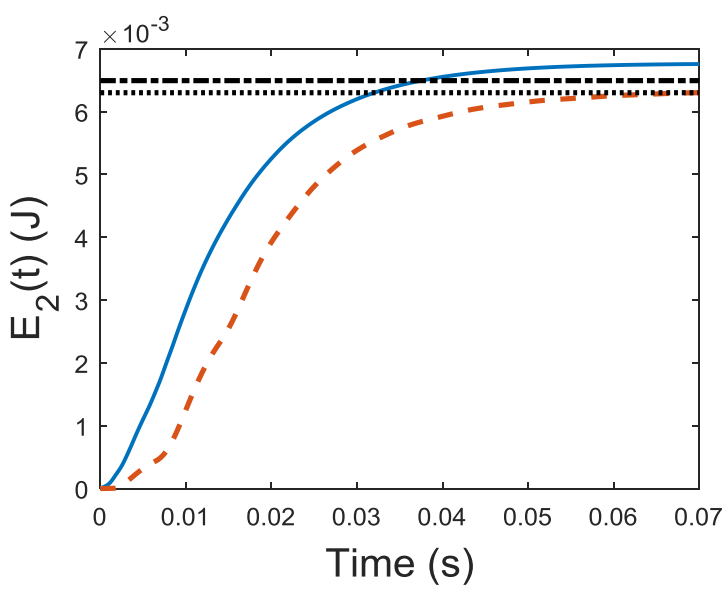

(b)

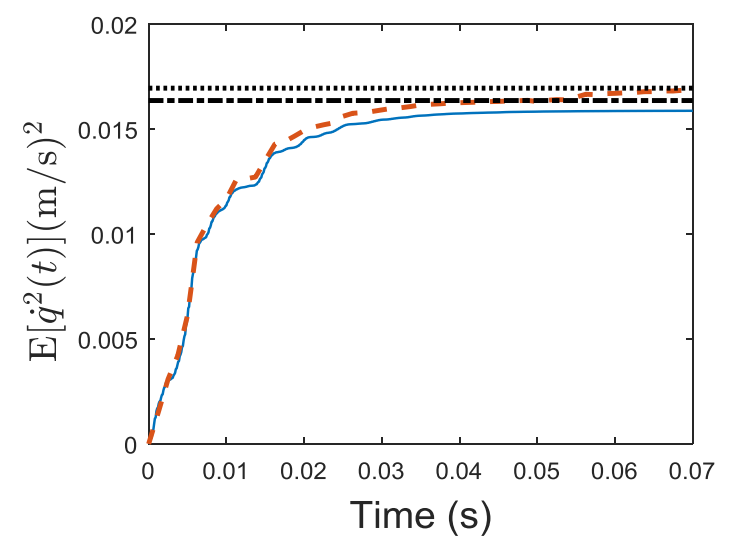

(c)

Figure 11. System response when a steady load is applied to a point on the beam and the system is initially at rest from simulations (solid) and transient hybrid method (dashed). Dash-dot and dotted lines represent response with steady-state excitation from simulations and the hybrid method of [4] respectively. (a) Plate one energy, (b) plate two energy and (c) mean square velocity of point on the beam. 


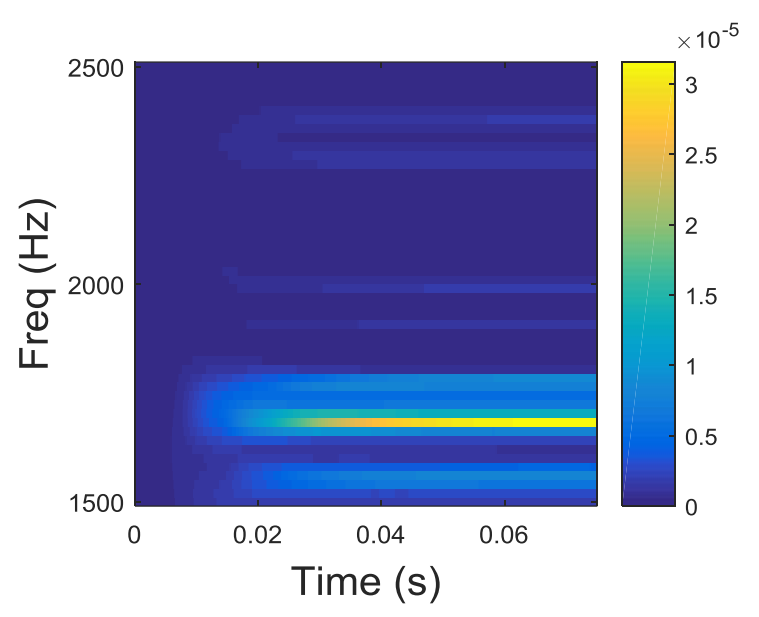

(a)

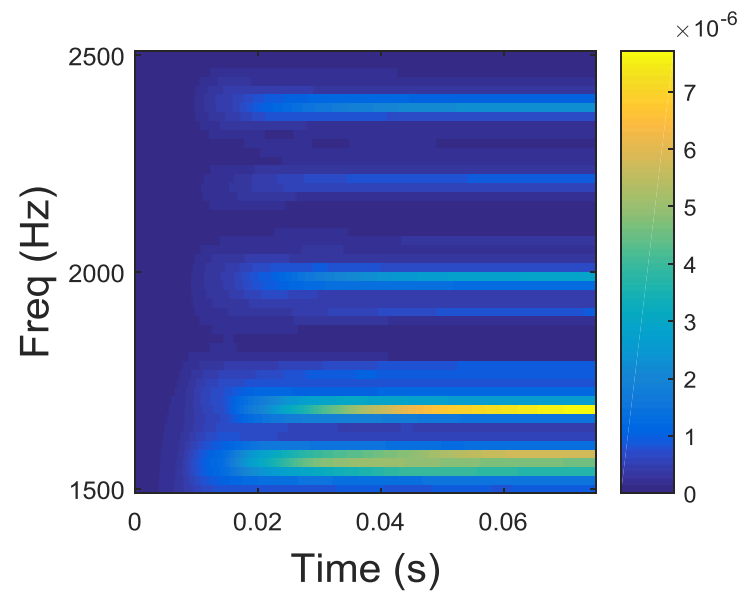

(b)

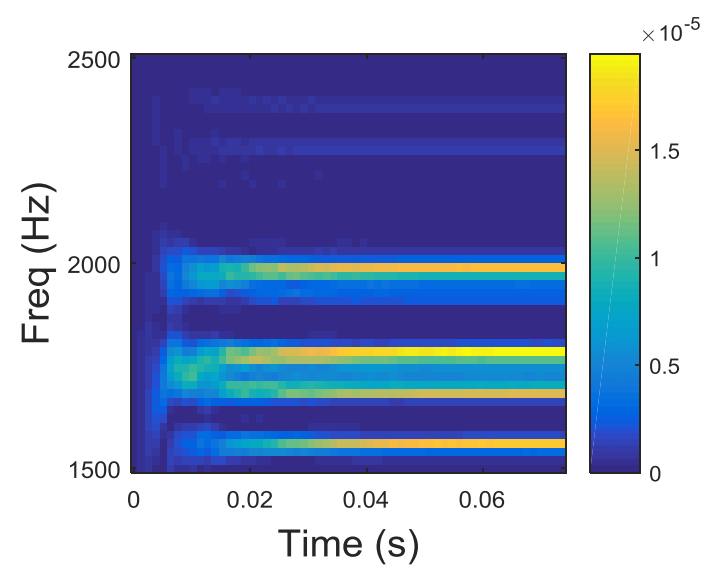

(c)

Figure 12. Evolutionary spectra from the transient hybrid FE-SEA method of (a) plate one energy, (b) plate two energy and (c) mean square velocity of a point on the beam when a steady load is applied to a point on the beam and the system is initially at rest.

\section{Conclusions}

The hybrid FE-SEA approach for modelling structures containing regions best characterised by both FE and SEA has been extended to predict the structural response under impulsive and time-varying excitation. Following the same approach as Shorter and Langley [4], a system can be split into components with only a few distinct natural frequencies, best modelled using FE analysis, and components with high statistical overlap, best modelled with SEA.

The Priestley formulation that encompasses the evolutionary spectrum of a random process has been used to represent both the deterministic degrees of freedom modelled with FE and the energies in 
the SEA subsystems. These two sets of variables can be related via the diffuse field reciprocity relationship [16,17]. However, since this result applies to structures under steady loading, an amended transient form that accounts for the time taken for a diffuse field to build up in a subsystem has been explored. It was found that the direct wavefield dominates the response at times shortly after an impulse, but as the field becomes more diffuse with time, the diffuse field reciprocity relationship begins to apply, and the subsystem reverberant forces can be accurately predicted.

A power balance equation, similar to the transient SEA equation, that investigates the power into each SEA subsystem has been derived and can be time-integrated to yield the time and frequency evolution of the subsystem energies and deterministic response. This method has been validated using FE simulations of a system consisting of two plates coupled by a beam and has shown the level of accuracy expected of an SEA-based approach in terms of both the energy in the SEA subsystems and the FE degrees of freedom as well as realistic qualitative time and frequency behaviour in the evolutionary spectra. However, the simulated results for the deterministic system exhibited oscillatory behaviour shortly after an impulse that was not predicted by the hybrid method. The oscillations are caused by the deterministic effects of the direct field in plates that are not included in the method. If greater accuracy is desired, a higher fidelity approach that incorporates the effects of both the direct and reverberant field could be devised.

\section{Acknowledgements}

The authors would like to thank Mitsubishi Heavy Industries for funding this research.

\section{Appendix: Reduction to steady-state hybrid FE-SEA method}

In this appendix, the steady response from the transient hybrid FE-SEA equations is shown to reduce to the response predicted by the steady-state hybrid FE-SEA method of [4] when a system is subjected to steady-state loading. Since [4] is concerned with dynamic stiffness matrices, denoted $\mathbf{D}$, the system mass, damping and stiffness matrices used in this paper can be combined by changing any differential operators to multiplications by $\mathrm{i} \omega$. When using dynamic stiffness matrices, the conversion to the statespace form of Eq. (18) is not required and to conform to the notation of [4] is not applied here.

The time dependence can be removed simply from the evolutionary spectrum formulation, such that $\mathbf{Q}(\omega, t) \rightarrow \mathbf{Q}(\omega)$. However, since the spectrum is Hermitian and double-sided this is not immediately equivalent to the frequency dependent terms used in the steady-state approach, denoted $\mathbf{q}_{\mathrm{ss}}(\omega)$, 
that generally represent the magnitude of a harmonic at a given frequency such that $\mathbf{q}(t)=$ $\mathbf{q}_{\mathrm{ss}}(\omega) \cos (\omega t+\epsilon)$, where $\epsilon$ is some phase. The two are related by $\mathbf{q}_{\mathrm{ss}}(\omega)=2 \mathbf{Q}(\omega)$ for $\omega>0$. In what follows, expressions for $\mathbf{Q}(\omega)$ will be found. Additionally, it should be noted that the incorporation of system and therefore response randomness is achieved in an equivalent manner through the ensemble averaging in both the evolutionary spectrum formulation and the steady-state approach.

In the steady-state case, a number of terms in the transient equations must be converted to their steady-state form for comparison with the equations of [4]. The following transformations take place

$$
\begin{gathered}
d^{(j)}(\omega, t) \rightarrow 1 \\
\mathbf{U}^{(j)}(\omega, t) \mathbf{U}^{(j) * \mathrm{~T}}(\omega, t) \rightarrow \frac{4 \alpha E_{j}(\omega)}{\omega \pi n_{j}} \operatorname{Im}\left[\mathbf{D}_{\mathrm{dir}}^{(j)}(\omega)\right] \\
\int_{-\infty}^{\infty} \mathbf{H}(\omega, t-\tau) a^{(j)}(\omega, \tau) \mathrm{e}^{-\mathrm{i} \omega(t-\tau)} \mathrm{d} \tau \rightarrow \mathrm{i} \omega \mathbf{D}_{\mathrm{tot}}^{-1}(\omega) .
\end{gathered}
$$

where the arrow represents both the collapse to steady-state form as well as from state-space to nodal coordinate representation. Equations (A.1) and (A.2) result because the wave field will be diffuse and not change with time under steady loading. The result of Eq. (A.3) follows since the integral yields the frequency response of the total deterministic system and the $\mathrm{i} \omega$ term is included because the velocities rather than displacements are of interest in the following power balance.

Evaluation of the power into a subsystem using Eq. (31) can now be made by combining the steadystate versions of Eqs. (32) and (33) to yield

$$
\begin{aligned}
p_{j}(\omega)=\sum_{m n} \operatorname{Im}\left[\mathbf{D}_{\text {dir }}^{(j)}\right]_{m n}\left\{\sum_{k}^{N} \frac{4 \alpha E_{k}}{\pi n_{k}} \mathbf{D}_{\text {tot }}^{-1} \operatorname{Im}\left[\mathbf{D}_{\text {dir }}^{(k)}\right] \mathbf{D}_{\mathrm{tot}}^{-\mathrm{H}}\right\}_{m n}+ \\
\sum_{m n} \operatorname{Im}\left[\mathbf{D}_{\mathrm{dir}}^{(j)}\right]_{m n}\left\{\mathbf{D}_{\mathrm{tot}}^{-1} \mathrm{~S}_{f f}^{\mathrm{ext}} \mathbf{D}_{\mathrm{tot}}^{-\mathrm{H}}\right\}_{m n}- \\
\frac{4 \alpha E_{j}}{\pi n_{j}} \sum_{m n} \operatorname{Im}\left[\mathbf{D}_{\mathrm{tot}}^{-1}\right]_{m n} \operatorname{Im}\left[\mathbf{D}_{\mathrm{dir}}^{(j)}\right]_{m n}
\end{aligned}
$$

where the arguments have been dropped and the identity $\sum_{m}\left\{\mathbf{A B}^{\mathrm{T}}\right\}_{m m} \equiv \sum_{m n} \mathbf{A}_{m n} \mathbf{B}_{m n}$ has been applied to the final term. The sum over the $N$ subsystems in the first term on the right-hand side can be expanded as

$$
\begin{aligned}
\sum_{k}^{N} \frac{4 \alpha E_{k}}{\pi n_{k}} \mathbf{D}_{\text {tot }}^{-1} \operatorname{Im}\left[\mathbf{D}_{\text {dir }}^{(k)}\right] \mathbf{D}_{\text {tot }}^{-\mathrm{H}}= & \sum_{k \neq j}^{N} \frac{4 \alpha E_{k}}{\pi n_{k}} \mathbf{D}_{\text {tot }}^{-1} \operatorname{Im}\left[\mathbf{D}_{\text {dir }}^{(k)}\right] \mathbf{D}_{\text {tot }}^{-\mathrm{H}}+ \\
& \frac{4 \alpha E_{j}}{\pi n_{j}} \mathbf{D}_{\text {tot }}^{-1} \operatorname{Im}\left[\mathbf{D}_{\text {tot }}-\mathbf{D}_{\mathrm{d}}-\sum_{k \neq j}^{N} \operatorname{Im}\left[\mathbf{D}_{\text {dir }}^{(k)}\right]\right] \mathbf{D}_{\text {tot }}^{-\mathrm{H}}
\end{aligned}
$$


where $\mathbf{D}_{\mathrm{d}}$ represents the dynamic stiffness of the deterministic system and a dynamic stiffness form of Eqs. (19) and (20) has been applied. Substituting Eq. (A.5) into Eq. (A.4) and noting that the final term in Eq. (A.4) cancels with the second term of Eq. (A.5) yields

$$
\begin{aligned}
p_{j}(\omega)= & \sum_{m n} \operatorname{Im}\left[\mathbf{D}_{\mathrm{dir}}^{(j)}\right]_{m n}\left\{\sum_{k \neq j}^{N} \frac{4 \alpha E_{k}}{\pi n_{k}} \mathbf{D}_{\mathrm{tot}}^{-1} \operatorname{Im}\left[\mathbf{D}_{\mathrm{dir}}^{(k)}\right] \mathbf{D}_{\mathrm{tot}}^{-\mathrm{H}}\right\}_{m n}- \\
& \sum_{m n} \frac{4 \alpha E_{j}}{\pi n_{j}} \operatorname{Im}\left[\mathbf{D}_{\mathrm{dir}}^{(j)}\right]_{m n}\left\{\sum_{k \neq j}^{N} \mathbf{D}_{\mathrm{tot}}^{-1} \operatorname{Im}\left[\mathbf{D}_{\mathrm{dir}}^{(k)}\right] \mathbf{D}_{\mathrm{tot}}^{-\mathrm{H}}\right\}_{m n}- \\
& \sum_{m n} \frac{4 \alpha E_{j}}{\pi n_{j}} \operatorname{Im}\left[\mathbf{D}_{\mathrm{dir}}^{(j)}\right]_{m n}\left\{\mathbf{D}_{\mathrm{tot}}^{-1} \operatorname{Im}\left[\mathbf{D}_{\mathrm{d}}\right] \mathbf{D}_{\mathrm{tot}}^{-\mathrm{H}}\right\}_{m n}+ \\
& \sum_{m n} \operatorname{Im}\left[\mathbf{D}_{\mathrm{dir}}^{(j)}\right]_{m n}\left\{\mathbf{D}_{\mathrm{tot}}^{-1} \mathrm{~S}_{f f}^{\mathrm{ext}} \mathbf{D}_{\mathrm{tot}}^{-\mathrm{H}}\right\}_{m n} .
\end{aligned}
$$

The first and second terms on the right-hand side of Eq. (A.6) represent respectively the power into the $j$ th subsystem from the reverberant fields of the other subystems and power from the reverberant field of the $j$ th subsystem to the other subsystems. The third term represents power lost from the reverberant field of the $j$ th subsystem to the damping of the deterministic system and the fourth term represents the power absorbed by the $j$ th subsystem from external excitation. These terms can be substituted into the power balance equation, Eq. (29), with the time derivative term set to zero and the result is identical to the power balance of [4] and thus the transient method has been shown to reduce to the steady-state method of [4]. Additionally, in the same way the steady-state hybrid method reduces to SEA, the transient hybrid method reduces to TSEA [13]. 


\section{References}

[1] R.H. Lyon, R.G. DeJong, Theory and application of statistical energy analysis, ButterworthHeinemann, Boston, 1995.

[2] V. Cotoni, P. Shorter, R. Langley, Numerical and experimental validation of a hybrid finite element-statistical energy analysis method, J. Acoust. Soc. Am. 122 (2007) 259-270. doi:10.1121/1.2739420.

[3] W. Desmet, B. Pluymers, O. Atak, CAE Methodologies for Mid-Frequency Analysis in Vibration and Acoustics, Katholieke Universiteit Leuven, Faculty of Engineering, Leuven, 2012.

[4] P.J. Shorter, R.S. Langley, Vibro-acoustic analysis of complex systems, J. Sound Vib. 288 (2005) 669-699. doi:10.1016/j.jsv.2005.07.010.

[5] J.E. Manning, K. Lee, Predicting mechanical shock transmission, Shock Vib. Bull. 37 (1968) 6570.

[6] R. Pinnington, D. Lednik, Transient statistical energy analysis of an impulsively excited two oscillator system, J. Sound Vib. 189 (1996) 249-264. doi:10.1006/jsvi.1996.0018.

[7] R.J. Pinnington, D. Lednik, Transient energy flow between two coupled beams, J. Sound Vib. 189 (1996) 265-287. doi:http://dx.doi.org/10.1006/jsvi.1996.0019.

[8] M.L. Lai, A. Soom, Statistical energy analysis for the time-integrated transient response of vibrating systems, J. Vib. Acoust. 112 (1990) 206-213. doi:10.1115/1.2930114.

[9] M.L. Lai, A. Soom, Prediction of transient vibration envelopes using statistical energy analysis techniques, J. Vib. Acoust. 112 (1990) 127-137. doi:10.1115/1.2930088.

[10] M. Robinson, C. Hopkins, Prediction of maximum time-weighted sound and vibration levels using transient statistical energy analysis. Part 1: Theory and numerical implementation, Acta Acust. United with Acust. 100 (2014) 46-56. doi:10.3813/AAA.918685.

[11] M. Robinson, C. Hopkins, Prediction of maximum time-weighted sound and vibration levels using transient statistical energy analysis. Part 2 : Experimental validation, Acta Acust. United with Acust. 100 (2014) 57-66. doi:10.3813/AAA.918686.

[12] C. Hopkins, M. Robinson, On the evaluation of decay curves to determine structural reverberation times for building elements, Acta Acust. United with Acust. 99 (2013) 226-244. doi:10.3813/AAA.918606. 
[13] R.S. Langley, D.H. Hawes, T. Butlin, Y. Ishii, A derivation of the Transient Statistical Energy Analysis (TSEA) equations with benchmark applications to plate systems, J. Sound Vib. "In Press. (2019).

[14] M.B. Priestley, Power spectral analysis of non-stationary random processes, J. Sound Vib. 6 (1967) 86-97. doi:10.1016/0022-460X(67)90160-5.

[15] R.S. Langley, On quasi-stationary approximations to non-stationary random vibration, J. Sound Vib. 113 (1987) 365-375. doi:10.1016/S0022-460X(87)80222-5.

[16] P.J. Shorter, R.S. Langley, On the reciprocity relationship between direct field radiation and diffuse reverberant loading, J. Acoust. Soc. Am. 117 (2005) 85-95. doi:10.1121/1.1810271.

[17] R.S. Langley, On the diffuse field reciprocity relationship and vibrational energy variance in a random subsystem at high frequencies, J. Acoust. Soc. Am. 121 (2007) 913-921. doi:10.1121/1.2409484.

[18] R.S. Langley, A transfer matrix analysis of the energetics of structural wave motion and harmonic vibration, Proc. R. Soc. London A. 452 (1996) 1631-1648.

[19] O. Guasch, C. García, Numerical local time stepping solutions for transient statistical energy analysis, J. Vib. Acoust. 136 (2014) 064502. doi:10.1115/1.4028454. 